\title{
3-D Semi-analytical model of a superconducting axial flux modulation machine
}

\author{
Rémi Dorget $^{1,2}$, Thierry Lubin ${ }^{2}$, Sabrina Ayat $^{1}$ and Jean Lévêque ${ }^{2}$ \\ ${ }^{1}$ Electrical \& Electronic Systems Research group, Safran Tech, 78114 Magny-Les-Hameaux, France \\ ${ }^{2}$ GREEN, Université de Lorraine, F-54000 Nancy, France
}

\begin{abstract}
This article presents a 3-D semi-analytical model of a partially superconducting axial flux modulation machine for the computation of the flux density distribution, the torque and the Eddy-current losses in the armature. The modulation of the superconducting coil magnetic field by the superconducting bulks is calculated by solving the Maxwell's equations in scalar potential with the variable separation method. Using Fourier series analysis and the convolution theorem, the bulk region is modelled by a domain with a spatially variable magnetic permeability. This variable permeability enables considering a diamagnetic bulk as well as a more complex magnetic behavior using the Bean model. The results of the proposed model are compared with 3-D finite elements models and good agreement is obtained for a significantly reduced computation time. The proposed model appears to be a suitable tool for optimisation and sizing processes.
\end{abstract}

Index Terms - Axial flux machine, high-temperature superconductivity, Semi-analytical model.

\section{INTRODUCTION}

The use of high temperature superconductors (HTS) in electrical machines is seen as a potential solution to enable aircraft electric propulsion [1]-[3]. To this end, HTS machines must reach a high specific power [4], recent studies have shown that power to mass ratios over $20 \mathrm{~kW} / \mathrm{kg}$ are achievable for MW-class generators and motors using liquid hydrogen as coolant [5]-[7]. Among the technologies considered, the flux modulation machine is a promising candidate as shown by several projects and articles on its radial flux form [8]-[10] and axial flux form [11], [12] including a recently built $50 \mathrm{~kW}$ prototype [13].

This last configuration, presented in Fig. 1, is composed of a static HTS coil fed with DC current generating a magnetic field in the machine. The rotor consists of several HTS bulks (one per pole pair) used in the superconducting mixed state. When the HTS bulks are subjected to the coil magnetic field, an electrical current is induced in the material to screen the magnetic field and the flux lines will concentrate in the apertures between the bulks. In the air gap, this flux concentration will generate a spatially variable magnetic field which generates an electromotive force in the two three-phases conventionnal armatures located on either side of the rotor when it rotates.

Due to the large magnetic flux that can be generated with HTS, no ferromagnetic material is required to reach a consequent magnetic loading, thus making this air-cored topology very light. The HTS coil is a static component, hence the machine magnetisation can be controlled from the coil without any slip rings. The absence of slip ring is a major advantage of this structure for reliability reason.

In order to properly exploit the potential of the flux modulation topology, an optimisation of the design must be performed and requires a precise and fast model. Numerical modelling using the finite element method (FEM) might be the most precise solution. However, the high computational cost of numerical method limits the number of parameter explored during the sizing process. Therefore, analytical and semianalytical modelling using the separation of variables method and Fourier series analysis can be used for the electromagnetic design of electrical machines [6], [14]. For common radial-flux structures such as induction machines, permanent magnet or wound rotor synchronous machines, cylindrical 2-D models are very efficient and have been extensively developed in the literature [15]-[18]. These topologies can also be modelled efficiently in 3-D [19].

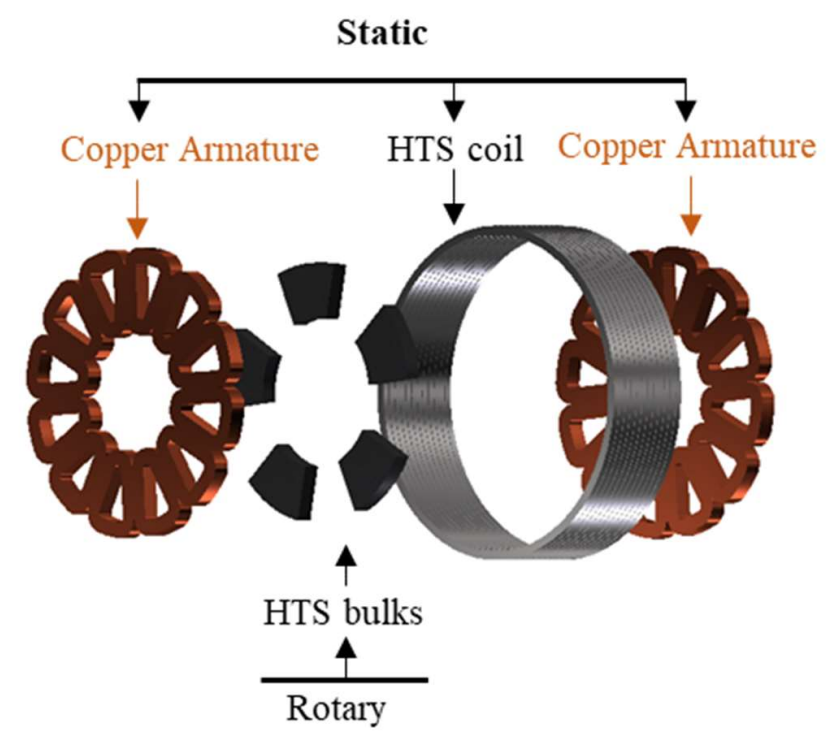

Fig. 1. Exploded view of the active elements in a partially superconducting axial flux modulation machine.

Nevertheless, axial flux machines do not have any symmetries that allow them to be accurately modelled in 2-D. Good results can be obtained under some assumptions to consider the end effects [20], though 3-D calculation is more suitable for that kind of problems. For the simulation of axial flux machines in 3-D there is typically two solutions, the first one being the use of a cylindrical frame which will lead to the 
most precise results but requires to manipulate Bessel functions [21]. A second option would be the use of a cartesian coordinate system by linearisation at the average radius symplifying the model by neglecting the curvature effects [22], [23]. These assumptions will be described in more details further in this article.

Moreover, flux modulation inductors have been analysed with analytical models, the radial flux topology has been solved in [10] while the axial flux topology with disk shaped bulks has been solved in [24]. In either case, the problem was adressed by superposing two 2-D models. The solution has been obtained by making the product of the magnetic field created by the superconducting coil(s) by a modulation function calculated by considering the bulks as perfectly diamagnetic domains. Both of these models have shown excellent results, but the perfect diamagnetism hypothesis is not necessarily verified in practice because it assumes an infinite or at least very high critical current density in the bulk [13], [25].

More recently, various research groups have developed models including subdomains with a spatially variable permeability, thus reducing the number of domains required to simulate machine with slots or variable reluctances [26]-[30]. These models are based on the convolution therorem that allows considering complex space distribution of the magnetic permeability in the subdomains. Thanks to this method, it is possible to simply take into account the saturation of the magnetic circuit [27], [29] and numerous radial-flux topologies have been modelled in recent years [26], [30].

A 3-D model of the axial flux modulation machine shown in Fig. 1 is presented in this paper. In section 2, the electromagnetic problem as well as the required assumptions are presented. In section 3 , the magnetic field distribution of the inductor is calculated with the variable separation method, Fourier series analysis and the convolution theorem for perfectly diamagnetic bulks. The section 4 presents the calculation of the machine power from the electromotive force as well as the eddy current losses in the armature. The results and the accuracy of this model will be discussed in the section 5. Finally, the model is improved in section 6 using the Bean model to consider the penetration of the magnetic field in the HTS bulks.

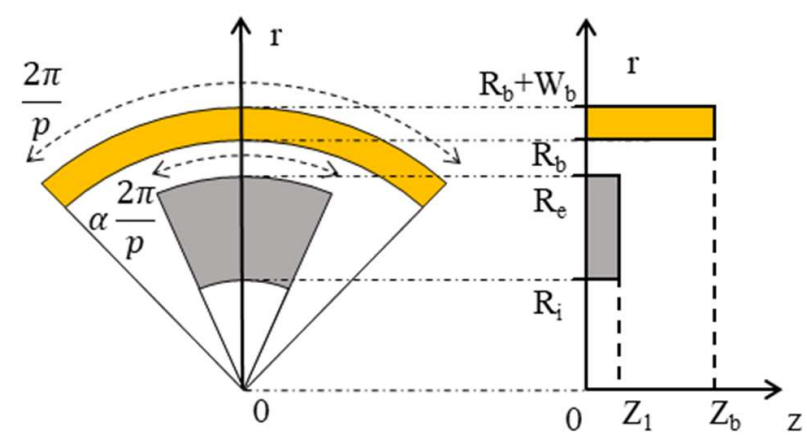

Fig. 2. Geometrical parameters of the inductor modelled parts according to the symmetries.

\section{PROBLEM DESCRIPTION}

The axial flux modulation inductor with ring segment shaped bulks has an inherent 3-D geometry and cannot be simply described in 2-D as was done for the axial flux inductor with round shaped bulk using two axisymmetric models [24]. Yet, some symmetries can still be exploited to simplify the problem. Indeed, due to the periodicity along azimuthal direction and to the symmetry in the axial direction, only half of a pole pair need to be considered. Thus, the geometrical problem to be solved according to the symmetries is presented in Fig. 2 with the geometrical parameters. The inner and outer radius of the bulk are $\mathrm{Ri}$ and Re, respectively. The inner radius of the coil is $\mathrm{R}_{b}$ and its thickness $\mathrm{Wb}$. The cylindrical frame is centred at the middle of the machine so $Z_{1}$ represent half of the bulk thickness and $Z_{b}$ half of the coil length. Finally, a bulk has an opening angle of $\alpha 2 \pi / p$ with $p$ being the number of pole pairs and $\alpha$ is the bulk pole-arc to pole-pitch ratio.

To simplify the resolution, the flux density distribution produced by the inductor will be obtained by separately calculating the magnetic field created by the coil and the deflection of the flux lines due to the bulks. The first problem is a 2-D axisymmetric model of the coil alone, which has already been described in [24] and will not be detailed in this paper. The second problem is a 3-D model of a bulk placed in a homogeneous external magnetic field from which a modulation function will be extracted. This modulation function represents the ability of the bulk to divert flux lines. Similar methods have already been applied in 2-D for various flux modulation machines [10], [24]. The calculation of the modulation function will be described in detail in this article. Ultimately, the actual flux density in the air gap is obtained by multiplying the magnetic field created by the coil by the modulation function.

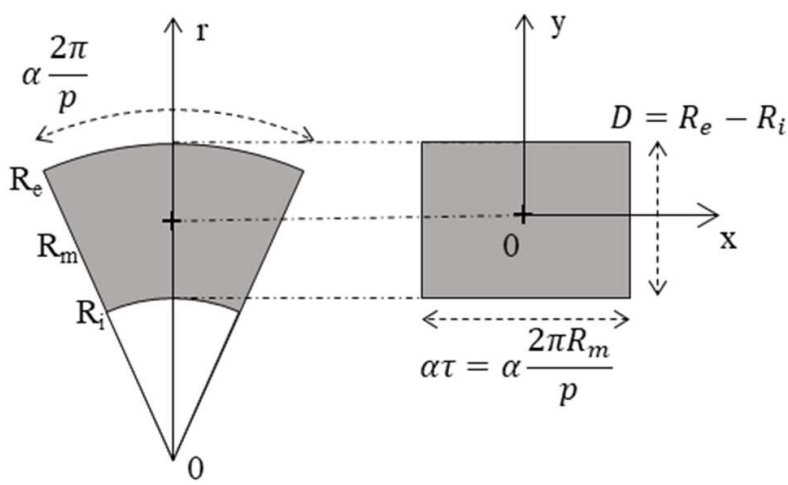

Fig. 3. Representation of the equivalent bulk geometry in the cartesian frame.

Considering the geometry, the more intuitive way to calculate the modulation function would be in a cylindrical coordinate system $(\mathrm{r}, \theta, \mathrm{z})$. However, it would lead to a complex system of differential equations to solve and requires the use of the Bessel functions. It has been observed in several references [22], [23], [31] that the curvature effects can be neglected by slightly changing the geometry from a cylindrical problem to a cartesian problem using a linearisation at the mean radius 
$R_{m}=\left(R_{e}+R_{i}\right) / 2$. Thus, the new geometry is presented in Fig. 3, the y-coordinate represents the radial direction while the $\mathrm{x}$-coordinate represents the azimuthal direction. The $\mathrm{x}$-coordinate is related to the $\theta$-coordinate by $x=R_{m} \theta$, the $\mathrm{y}$-coordinate is related to the r-coordinate by $y=r-R_{m}$ and the $\mathrm{z}$-coordinate is identical for both frames. The height of the rectangle shaped bulk is $D=R_{e}-R_{i}$ and its length is $\alpha \tau, \tau=$ $\frac{2 \pi}{p} R_{m}$ being the length of a pole pair. The impact of these assumptions on the model precision will be discussed in section 5 .

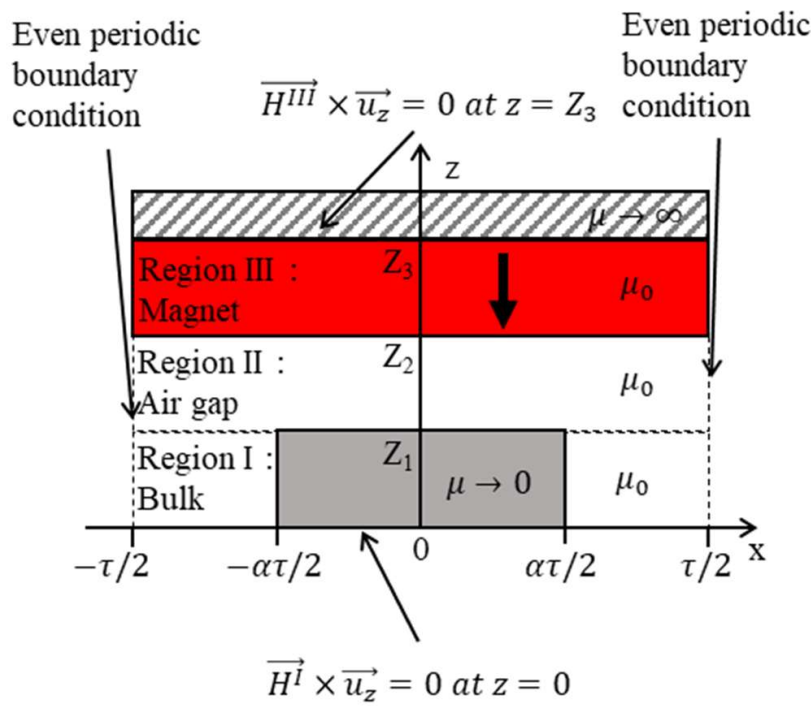

Fig. 4. Sectional view of the cartesian problem in the (xz) plane with the different regions and boundary conditions.

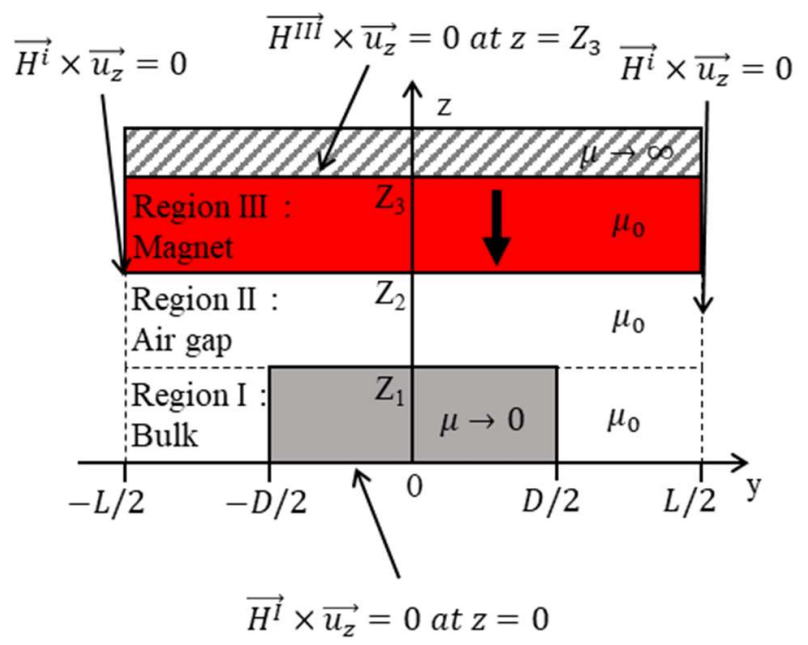

Fig. 5. Sectional view of the cartesian problem in the (yz) plane with the different regions and boundary conditions.

\section{III. . 3-D SEMI-ANALYTICAL MODEL}

The cross-sectional views of the cartesian problem for the modulation function are shown in Fig. 4 and Fig. 5 in planes (xz) and (yz) respectively. The external homogeneous magnetic field will be modelled here by a permanent magnet placed on a ferromagnetic plate with an assumed infinite permeability creating a magnetic field along the z-axis which is modulated by the HTS shield. The iron plate and the magnet must be located far enough from the bulk surface otherwise the plate disturbs the magnetic field distribution and the result would not correspond to the modulation of a homogeneous field. This distance has been set at $Z_{2}=5 R_{e}$ to avoid such issues. The $\mathrm{x}$ and $\mathrm{z}$ components of the modulation function will be obtained by dividing the $\mathrm{x}$ and $\mathrm{z}$ components of the flux density in the presence of the bulk with the z-component of the flux density in the absence of the bulk. The ferromagnetic plate and the magnet surface are at a distance $Z_{3}$ and $Z_{2}$ from the bulk centre respectively. The magnet remanent magnetization is written $B_{\text {rem }}$ and the magnetization $\vec{M}$ is in the opposite direction to the z-axis, $\vec{M}=-B_{\text {rem }} / \mu_{0} \overrightarrow{u_{z}}$.

It should be noted that the uniform applied magnetic field could have been modelled by applying a Dirichlet condition on the scalar potential on the boundary at $Z_{2}$. However, it has been decided to model it through a magnet for the sake of understanding.

In this section, the bulk is considered as an almost perfect diamagnetic material with a relative permeability $\mu_{r}=10^{-4}$ while the other parts of the problem have a relative permeability equal to 1 . The whole geometry is divided into three regions:

The HTS bulk region (region I)

The air gap region (region II)

The magnet region (region III)

Since there is no current in any of the regions, $\vec{H}^{i}$, the magnetic field in region $i$, will be expressed in term of a magnetic scalar potential $\Phi^{\mathrm{i}}$, which is defined as:

$$
\vec{H}^{i}=-\vec{\nabla} \times \Phi^{i}(i=I, I I, I I I)
$$

\section{A. Boundary conditions}

Solving this problem requires the definitions of boundary conditions. The boundaries on the $\mathrm{x}$-direction and $\mathrm{y}$-direction will define the Sturm-Liouville problem eigenvalues and define the general solution form while the boundary and interface conditions in the z-direction will be used to calculate the unknown coefficients.

Due to the bulks periodicity in the x-direction, the magnetic problem presents even boundary conditions in the $\mathrm{x}$-direction. Thus, the domains are limited to $x \in[-\tau / 2 ; \tau / 2]$, and the boundary conditions are:

$$
\Phi^{i}(-\tau / 2, y, z)=\Phi^{i}(\tau / 2, y, z)
$$

The conditions in the y-direction appear less naturally. Indeed, far enough from its surface, the bulk has no more influence on the magnetic field. Hence, we define the limits of the domain in the y-direction as $-L / 2$ and $L / 2$ on which the boundary conditions are imposed as:

$$
\Phi^{i}(x,-L / 2, z)=\Phi^{i}(x, L / 2, z)=0
$$

The value of $\mathrm{L}$, which is the domain size, must be chosen 
carefully. If the domain limits are too close to the bulk, the boundaries will have a significant impact on the result and will lead to errors. However, if $L$ is too large, too many harmonics would be required to solve the problem, thus increasing the computational cost of the model. For the rest of the article, the value $L=3 D$ has been chosen after trials and errors.

Eventually, in the z-direction, the ferromagnetic plate and the axial symmetry in $z=Z_{3}$ and $z=0$, respectively, lead to the orthogonality of the magnetic field for both cases that expressed in scalar potential give:

$$
\begin{gathered}
\Phi^{I}(x, y, 0)=0 \\
\Phi^{I I I}\left(x, y, z_{3}\right)=0
\end{gathered}
$$

\section{B. Separation of variables and Fourier series representation}

The magnetic scalar potential solution in each domain is obtained using the separation of variables. Considering the boundary conditions in the $\mathrm{x}$ and $\mathrm{y}$ directions, $\Phi^{\mathrm{i}}$ is expressed in terms of 2-D complex Fourier series:

$$
\Phi^{i}(x, y, z)=\sum_{n=-N}^{N} \sum_{k=-N}^{N} \hat{\varphi}_{n, k}^{i}(z) e^{j \frac{2 \pi n}{\tau} x} e^{j \frac{2 \pi k}{L} y}
$$

The harmonics ranks are denoted by $n$ and $k$ for the $\mathrm{x}$ and $\mathrm{y}$ directions respectively. $N$ represents the highest spatial harmonic considered in both directions. Ideally, the value of $\mathrm{N}$ should be infinite. In practice, it is selected to satisfy the balance between accuracy and computational cost. The impact of $N$ on the computation time and model precision will be discussed further. Similarly the magnetic field $\vec{H}^{i}$ components are also expressed as Fourier series:

$$
\begin{aligned}
& \vec{H}^{i}=H_{x}^{i} \overrightarrow{u_{x}}+H_{y}^{i} \overrightarrow{u_{y}}+H_{z}^{i} \overrightarrow{u_{z}} \\
& H_{x}^{i}(x, y, z) \\
& =\sum_{n=-N}^{N} \sum_{k=-N}^{N} \widehat{H}_{x_{n, k}}^{i}(z) e^{j \frac{2 \pi n}{\tau} x} e^{j \frac{2 \pi k}{L} y} \\
& H_{y}^{i}(x, y, z) \\
& =\sum_{n=-N}^{N} \sum_{k=-N}^{N} \widehat{H}_{y_{n, k}}^{i}(z) e^{j \frac{2 \pi}{\tau} x} e^{j \frac{2 \pi k}{L} y} \\
& H_{z}^{i}(x, y, z) \\
& =\sum_{n=-N}^{N} \sum_{k=-N}^{N} \widehat{H}_{z, k}^{i}(z) e^{j \frac{2 \pi}{\tau} x} e^{j \frac{2 \pi k}{L} y}
\end{aligned}
$$

Whereas the flux density $\overrightarrow{B^{\imath}}$ components are expressed as:

$$
\begin{gathered}
\overrightarrow{B^{\imath}}=B_{x}^{i} \overrightarrow{u_{x}}+B_{y}^{i} \overrightarrow{u_{y}}+B_{z}^{i} \overrightarrow{u_{z}} \\
B_{x}^{i}(x, y, z) \\
=\sum_{n=-N}^{N} \sum_{k=-N}^{N} \hat{B}_{x_{n, k}}^{i}(z) e^{j \frac{2 \pi n}{\tau} x} e^{j \frac{2 \pi k}{L} y} \\
B_{y}^{i}(x, y, z) \\
=\sum_{n=-N}^{N} \sum_{k=-N}^{N} \hat{B}_{y_{n, k}}^{i}(z) e^{j \frac{2 \pi n}{\tau} x} e^{j \frac{2 \pi k}{L} y}
\end{gathered}
$$

$$
\begin{aligned}
& B_{z}^{i}(x, y, z) \\
& =\sum_{n=-N}^{N} \sum_{k=-N}^{N} \hat{B}_{z, k}^{i}(z) e^{j \frac{2 \pi n}{\tau} x} e^{j \frac{2 \pi k}{L} y}
\end{aligned}
$$

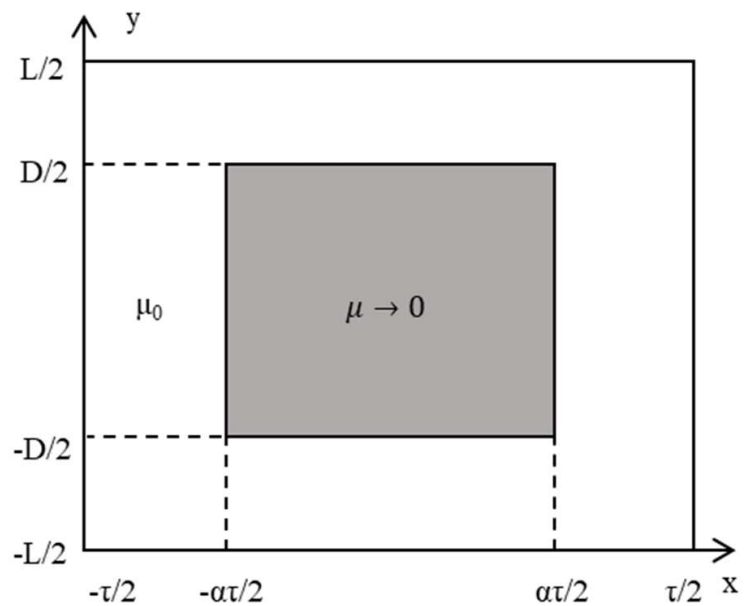

Fig. 6. Magnetic permeability distribution in the region I over the (xy) plane.

Thanks to the Fourier series representation, only the $z$-dependant coefficients of the scalar potential $\hat{\varphi}_{n, k}^{i}(z)$, the magnetic field components $\widehat{H}_{x_{n, k}}^{i}(z), \widehat{H}_{y_{n, k}}^{i}(z), \widehat{H}_{z_{n, k}}^{i}(z)$ and the flux density components $\hat{B}_{x_{n, k}}^{i}(z), \hat{B}_{y_{n, k}}^{i}(z), \hat{B}_{z_{n, k}}^{i}(z)$ remain to be determined.

\section{Convolution theorem}

The magnetic permeability is homogeneous in the regions II and III and equal to $\mu_{0}$. However in the region I, the permeability is equal to $\mu_{r} \mu_{0}$ with $\mu_{r}=10^{-4}$ at the bulk location and $\mu_{0}$ elsewhere as shown in Fig. 6 . The permability in the region I is inhomogeneous and must be decomposed in Fourier series as well:

$$
\mu^{I}(x, y)=\sum_{n=-N}^{N} \sum_{k=-N}^{N} \hat{\mu}_{n, k} e^{j \frac{2 \pi n}{\tau} x} e^{j \frac{2 \pi k}{L} y}
$$

Where the coefficients $\hat{\mu}_{n, k}$ are expressed as:

$$
\hat{\mu}_{n, k}=\left\{\begin{array}{c}
\mu_{0}\left(1+\alpha \frac{D}{L}\left(\mu_{r}-1\right)\right), \\
\text { for } n=k=0 \\
\frac{\mu_{0} D}{\pi n L}\left(\mu_{r}-1\right) \sin (n \pi \alpha), \\
\text { for } n \neq 0 \& k=0 \\
\frac{\mu_{0} \alpha}{\pi k}\left(\mu_{r}-1\right) \sin \left(k \pi \frac{D}{L}\right), \\
\text { for } n=0 \& k \neq 0 \\
\frac{\mu_{0}}{\pi n k}\left(\mu_{r}-1\right) \sin (n \pi \alpha) \sin \left(k \pi \frac{D}{L}\right), \\
\text { for } n \neq 0 \& k \neq 0
\end{array}\right.
$$


It should be noted here that the bulk relative permeability $\mu_{r}$ cannot be equal to zero as the resolution of the magnetostatic problem will involve the inverse of the permeability. Therefore, the permeability value must be small enough to behave almost as a perfect diamagnetic material. Conversely, a too small value of $\mu_{r}$ would cause numerical problems. Thus, the value $\mu_{r}=$ $10^{-4}$ has been chosen after trials.

The constitutive relation between $\vec{B}$ and $\vec{H}$ in nonmagnetized regions is:

$$
\overrightarrow{B^{I}}=\mu^{I}(x, y) \overrightarrow{H^{I}}
$$

The right part of (17) being the product of two Fourier series decomposition, the convolution theorem can be applied allowing to express the coefficients of each component of $\vec{B}$ as follows:

$$
\begin{aligned}
& \widehat{B}_{x_{n, k}}^{I}(z)=\sum_{n^{\prime}=-N} \sum_{k^{\prime}=-N}^{N} \hat{\mu}_{n-n^{\prime}, k-k^{\prime}} \widehat{H}_{x_{n^{\prime}, k}^{\prime}}(z) \\
& \widehat{B}_{n, k}^{I}(z)=\sum_{n^{\prime}=-N} \sum_{k^{\prime}=-N}^{N} \hat{\mu}_{n-n^{\prime}, k-k^{\prime}} \widehat{H}_{y_{n^{\prime}, k \prime}^{I}}(z) \\
& \widehat{B}_{z, k}^{I}(z)=\sum_{n^{\prime}=-N} \sum_{k^{\prime}=-N}^{N} \hat{\mu}_{n-n^{\prime}, k-k^{\prime}} \widehat{H}_{z}^{I}
\end{aligned}
$$

It can be seen from equations (18) to (20) that each harmonic of $\vec{B}$ depends on all harmonics of $\vec{H}$, whereas for homogeneous permeability regions, only harmonics of the same rank are interdependent. Hence, the application of Maxwell's equations in this region leads to a system of differential equations to solve.

\section{Matrix form}

In order to solve the system of differential equations, (18), (19) and (20) are rewritten in matrix form:

$$
\begin{aligned}
B_{x}^{I} & =\boldsymbol{\mu}_{c}^{I} \boldsymbol{H}_{x}^{I} \\
\boldsymbol{B}_{\boldsymbol{y}}^{I} & =\boldsymbol{\mu}_{c}^{I} \boldsymbol{H}_{y}^{I} \\
\boldsymbol{B}_{z}^{I} & =\boldsymbol{\mu}_{c}^{I} \boldsymbol{H}_{z}^{I}
\end{aligned}
$$

In matrix form, every quantity $X(x, y, z)$ expressed as Fourier series $\left(B_{x}, B_{y}, B_{z}, H_{x}, H_{y}, H_{z}\right.$ and $\left.\Phi\right)$ with coefficients $\widehat{X_{n, k}}(z)$ can be represented in matrix form by gathering the coefficient in a column vector $\boldsymbol{X}$ as follows:

$$
\boldsymbol{X}=X_{m}=\widehat{X_{n, k}}(z)
$$

The relations between the vector element index $m$ and the harmonic ranks $n$ and $k$ are:

$$
\begin{gathered}
k=f i x\left(\frac{m-1}{2 N+1}\right)-N \\
n=\bmod (m-1,2 N+1)-N
\end{gathered}
$$

The functions $f i x(u)$ and $\bmod (u, v)$ are the rounding toward zero of $\mathrm{u}$ and the remainder of the division of $u$ and $v$, respectively.

The matrix $\boldsymbol{\mu}_{c}^{I}$ is the permeability convolution matrix and is defined according to (18), (19) and (20):

$$
\boldsymbol{\mu}_{c}^{I}=\mu_{c m, l}^{I}=\hat{\mu}_{n-n^{\prime}, k-k^{\prime}}
$$

The relations between the matrix element indexes $\mathrm{m}, 1$ and the harmonic ranks $\mathrm{n}, \mathrm{k}, \mathrm{n}$ ', k' are:

$$
\begin{gathered}
k=f i x\left(\frac{m-1}{2 N+1}\right)-N \\
n=\bmod (m-1,2 N+1)-N \\
k^{\prime}=f i x\left(\frac{l-1}{2 N+1}\right)-N \\
n^{\prime}=\bmod (l-1,2 N+1)-N
\end{gathered}
$$

In the regions II and III, the matrix form will also be used although the permeability convolution matrixes $\boldsymbol{\mu}_{c}^{I I}$ and $\boldsymbol{\mu}_{c}^{I I I}$ are much simpler:

$$
\boldsymbol{\mu}_{c}^{I I}=\boldsymbol{\mu}_{c}^{I I I}=\mu_{0} \boldsymbol{I}_{(\mathbf{2 N}+\mathbf{1})^{2}}
$$

With $\boldsymbol{I}_{(\mathbf{2 N + 1})^{2}}$ the $(2 N+1)^{2}$ by $(2 N+1)^{2}$ identity matrix.

\section{E. Magnetostatic field equation}

Using the matrix form described above, the relation (1) becomes:

$$
\begin{gathered}
\boldsymbol{H}_{x}^{i}=-j \frac{2 \pi}{\tau} \boldsymbol{N}_{x} \Phi^{i} \\
\boldsymbol{H}_{\boldsymbol{y}}^{i}=-j \frac{2 \pi}{L} \boldsymbol{N}_{\boldsymbol{y}} \boldsymbol{\Phi}^{i} \\
\boldsymbol{H}_{z}^{i}=-\frac{\partial \boldsymbol{\Phi}^{i}}{\partial z}
\end{gathered}
$$

With the matrices $\mathbf{N}_{\mathbf{x}}$ and $\mathbf{N}_{\mathbf{y}}$ being defined as:

$$
\begin{gathered}
\boldsymbol{N}_{\boldsymbol{x}}=N_{x_{m, l}}=\left\{\begin{array}{c}
\bmod (m-1,2 N+1)-N \\
\text { for } m=l \\
0 \text { for } m \neq l
\end{array}\right. \\
\boldsymbol{N}_{\boldsymbol{y}}=N_{y_{m, l}}=\left\{\begin{array}{c}
\text { fix }\left(\frac{m-1}{2 N+1}\right)-N \\
\text { for } m=l \\
0 \text { for } m \neq l
\end{array}\right.
\end{gathered}
$$

The magnetostatic field equation in scalar potential is then derived from the Gauss law for magnetism:

$$
\nabla \cdot \vec{B}^{i}=0
$$

Substituting (21), (22) and (23) into (38) leads to the following equation since the divergence of the magnetization is equal to zero in each region:

$$
\boldsymbol{\mu}_{c}^{i} \frac{\partial \boldsymbol{H}_{z}^{i}}{\partial z}+j \frac{2 \pi}{\tau} \boldsymbol{N}_{x} \boldsymbol{\mu}_{c}^{i} \boldsymbol{H}_{x}^{I}+j \frac{2 \pi}{L} \boldsymbol{N}_{y} \boldsymbol{\mu}_{c}^{i} \boldsymbol{H}_{y}^{I}=0
$$

Finally, the magnetostatic equation to solve in scalar potential is obtained by substituting (33), (34) and (35) in (39):

$$
\frac{\partial^{2} \boldsymbol{\Phi}^{i}}{\partial z^{2}}-\left(\boldsymbol{V}^{i}\right)^{2} \boldsymbol{\Phi}^{i}=0
$$

Where in the region I:

$$
\begin{gathered}
\boldsymbol{V}^{I}=\left(\left(\frac{2 \pi}{\tau}\right)^{2}\left(\boldsymbol{\mu}_{c}^{I}\right)^{-1} \boldsymbol{N}_{x} \boldsymbol{\mu}_{c}^{I} \boldsymbol{N}_{\boldsymbol{x}}+\right. \\
\left.\left(\frac{2 \pi}{L}\right)^{2}\left(\boldsymbol{\mu}_{c}^{I}\right)^{-1} \boldsymbol{N}_{\boldsymbol{y}} \boldsymbol{\mu}_{c}^{I} \boldsymbol{N}_{\boldsymbol{y}}\right)^{\frac{1}{2}}
\end{gathered}
$$

Whereas in the region II and III, the matrix V is diagonal: 


$$
\boldsymbol{V}^{I I}=\boldsymbol{V}^{I I I}=\left(\left(\frac{2 \pi}{\tau}\right)^{2}\left(\boldsymbol{N}_{x}\right)^{2}+\left(\frac{2 \pi}{L}\right)^{2}\left(N_{y}\right)^{2}\right)^{\frac{1}{2}}
$$

\section{F. General solution to the magnetostatic equation}

In each region $i$, the general solution of (40) is:

$$
\boldsymbol{\Phi}^{i}=e^{V^{i} z} C_{1}^{i}+e^{-V^{i} z} C_{2}^{i}
$$

Where $\boldsymbol{C}_{\mathbf{1}}^{\boldsymbol{i}}$ and $\boldsymbol{C}_{2}^{\boldsymbol{i}}$ are column vectors with unknown coefficients of each region. In order to evaluate the matrix exponentials involved in (43), eigendecomposition is applied to $e^{V^{i} z}$ :

$$
e^{V^{i} z}=\boldsymbol{P}^{i} e^{D^{i} z}\left(\boldsymbol{P}^{i}\right)^{-1}
$$

Where $\boldsymbol{D}^{i}$ and $\boldsymbol{P}^{\boldsymbol{i}}$ are the diagonal eigenvalue matrix and the eigenvector matrix of $\boldsymbol{V}^{i}$, respectively. As a result, the general solution (43) becomes:

$$
\boldsymbol{\Phi}^{i}=\boldsymbol{P}^{i} e^{D^{i} z}\left(\boldsymbol{P}^{i}\right)^{-1} C_{1}^{i}+\boldsymbol{P}^{i} e^{-D^{i} z}\left(\boldsymbol{P}^{i}\right)^{-1} C_{2}^{i}
$$

As $V^{I I}$ and $\boldsymbol{V}^{I I I}$ are diagonal matrices, their eigenvector matrices are equal to the identity matrix $I_{(2 N+1)}{ }^{2}$ and $\boldsymbol{V}^{I I}, \boldsymbol{V}^{I I I}, \boldsymbol{D}^{I I}$ and $\boldsymbol{D}^{I I I}$ are equal.

\section{G. Calculation of the coefficients}

The determination of the six vectors of coefficients of the general solution will require the resolution of a system of linear equations and the inversion of a $6(2 N+1)^{2}$ by $6(2 N+1)^{2}$ matrix [28]. Thus, for $N=10$, the solution will involve the inversion of a 2646 by 2646 matrix which would have an important computational cost. Nevertheless, it is possible to drastically reduce the size of the linear system by rewriting (45) according to the boundary conditions in the z-direction.

In matrix form, the boundary conditions (4) and (5) as well as the conditions between each domains lead to the following equations:

$$
\begin{gathered}
\left.\boldsymbol{\Phi}^{I}\right|_{z=0}=\mathbf{0} \\
\left.\boldsymbol{\Phi}^{I}\right|_{z=Z_{1}}=\left.\boldsymbol{\Phi}^{I I}\right|_{z=Z_{1}} \\
\left.\boldsymbol{\mu}_{c}^{I} \frac{\partial \boldsymbol{\Phi}^{I}}{\partial z}\right|_{z=z_{1}}=\left.\mu_{0} \frac{\partial \boldsymbol{\Phi}^{I I}}{\partial z}\right|_{z=z_{1}} \\
\frac{\partial \boldsymbol{\Phi}^{I I I}}{\partial z}-\left.\frac{\partial \boldsymbol{\Phi}^{I I}}{\partial z}\right|_{z=Z_{2}}=\left.\boldsymbol{\Phi}^{I I I}\right|_{z=Z_{2}}=M^{I I I} \\
\left.\boldsymbol{\Phi}^{I I I}\right|_{z=Z_{3}}=\mathbf{0}
\end{gathered}
$$

Where $\boldsymbol{M}^{I I I}$ is the vector representing the magnetization in the region III expressed as:

$$
\boldsymbol{M}^{I I I}=M_{m}^{I I I}=\left\{\begin{array}{cl}
\frac{-B_{r e m}}{\mu_{0}}, & m=2 N^{2}+2 N+1 \\
0 & , \text { otherwise }
\end{array}\right.
$$

In the region I, according to the conditions (46) and (47) and to the superposition theorem, the general solution can be rewritten as:

$$
\boldsymbol{\Phi}^{I}=\boldsymbol{P}^{I} \frac{\sinh \left(\boldsymbol{D}^{I} z\right)}{\sinh \left(\boldsymbol{D}^{I} Z_{1}\right)}\left(\boldsymbol{P}^{I}\right)^{-\mathbf{1}} \boldsymbol{a}^{I}
$$

In the region II, according to the conditions (47) and (48):

$$
\begin{aligned}
\boldsymbol{\Phi}^{I I}=\frac{\sinh \left(\boldsymbol{D}^{I I}\left(z-Z_{2}\right)\right)}{\sinh \left(\boldsymbol{D}^{I I}\left(Z_{1}-Z_{2}\right)\right)} \boldsymbol{a}^{I I} & \\
& +\frac{\sinh \left(\boldsymbol{D}^{I I}\left(z-Z_{1}\right)\right)}{\sinh \left(\boldsymbol{D}^{I I}\left(Z_{2}-Z_{1}\right)\right)} \boldsymbol{b}^{I I}
\end{aligned}
$$

Eventually, in the region III, according to (49) and (51):

$$
\boldsymbol{\Phi}^{I I I}=\frac{\sinh \left(\boldsymbol{D}^{I I I}\left(z-Z_{3}\right)\right)}{\sinh \left(\boldsymbol{D}^{I I I}\left(Z_{2}-Z_{3}\right)\right)} \boldsymbol{b}^{I I I}
$$

The function $\sinh (\boldsymbol{X})$ is the hyperbolic sine of the matrix $\boldsymbol{X}$ and defined similarly to the hyperbolic sinus for scalar values. The function hyperbolic cosine of the matrix $\boldsymbol{X} \cosh (\boldsymbol{X})$ can also be defined.

Thanks to the reformulations of the general solutions, the relations (53), (54) and (55) show only 4 coefficients vectors to be determined, $\boldsymbol{a}^{I}, \boldsymbol{a}^{I I}, \boldsymbol{b}^{I I}$ and $\boldsymbol{b}^{I I I}$. Furthermore, substituting (53) and (54) into (47) results in the relation:

$$
\boldsymbol{a}^{I}=\boldsymbol{a}^{I I}
$$

In a similar way, (54) and (55) into (49) lead to:

$$
\boldsymbol{b}^{I I I}=\boldsymbol{b}^{I I}
$$

As a result, there are only two coefficients vectors remaining. Ultimately, the conditions (48) and (50) are used to calculate $\boldsymbol{a}^{I I}$ and $\boldsymbol{b}^{I I}$. These two relations are expressed as the following system:

$$
\left[\begin{array}{ll}
\boldsymbol{E}_{11} & \boldsymbol{E}_{12} \\
\boldsymbol{E}_{21} & \boldsymbol{E}_{22}
\end{array}\right]\left(\begin{array}{l}
\boldsymbol{a}^{I I} \\
\boldsymbol{b}^{I I}
\end{array}\right)=\left(\begin{array}{c}
\mathbf{0} \\
\left(\boldsymbol{D}^{I I I}\right)^{-1} \boldsymbol{M}^{I I I}
\end{array}\right)
$$

Where the component of the matrix $\boldsymbol{E}$ are:

$$
\begin{gathered}
\boldsymbol{E}_{11}=\boldsymbol{\mu}_{c}^{I} \boldsymbol{P}^{I} \boldsymbol{D}^{I} \frac{\cosh \left(\boldsymbol{D}^{I} Z_{1}\right)}{\sinh \left(\boldsymbol{D}^{I} Z_{1}\right)}\left(\boldsymbol{P}^{\boldsymbol{I}}\right)^{-\mathbf{1}} \\
-\mu_{0} \boldsymbol{D}^{I I} \frac{\cosh \left(\boldsymbol{D}^{I I}\left(Z_{1}-Z_{2}\right)\right)}{\sinh \left(\boldsymbol{D}^{I I}\left(Z_{1}-Z_{2}\right)\right)} \\
\boldsymbol{E}_{12}=-\mu_{0} \boldsymbol{D}^{I I} \frac{\boldsymbol{I}_{(\mathbf{2 N + 1})^{2}}}{\sinh \left(\boldsymbol{D}^{I I}\left(Z_{2}-Z_{1}\right)\right)} \\
\boldsymbol{E}_{\mathbf{2 1}}=-\frac{\boldsymbol{I}_{(\mathbf{2 N}+\mathbf{1})^{\mathbf{2}}}}{\sinh \left(\boldsymbol{D}^{I I}\left(Z_{1}-Z_{2}\right)\right)} \\
\boldsymbol{E}_{\mathbf{2 2}}=\frac{\cosh \left(\boldsymbol{D}^{I I I}\left(Z_{2}-Z_{3}\right)\right)}{\sinh \left(\boldsymbol{D}^{I I I}\left(Z_{2}-Z_{3}\right)\right)} \\
-\frac{\cosh \left(\boldsymbol{D}^{I I}\left(Z_{2}-Z_{1}\right)\right)}{\sinh \left(\boldsymbol{D}^{I I}\left(Z_{2}-Z_{1}\right)\right)}
\end{gathered}
$$

The linear system $(58)$ has $2(2 N+1)^{2}$ unknown coefficients and requires, for $N=10$, the inversion of only a 882 by 882 matrix. It should be noted that the zero harmonics cause the matrix $\boldsymbol{E}$ to be non-invertible since the solution (43) is only valid for non-zero harmonics. To overcome this issue, a very small value is given to the zero harmonics such as $10^{-5}$ instead of absolute zero [26].

\section{H. Calculation of the flux modulation function}

The flux modulation function is to be calculated in the region II as it corresponds to the location of the airgap and the armature. In order to compute the torque, only the axial component of the magnetic flux density is required while the eddy current losses in the windings involve the tangential component in the $\theta$-direction as well. Thus, in the cartesian frame, only the x-component and z-component of the magnetic field in the region II have to be calculated. As the magnetic 
scalar potential is now known in each region, the vectors $\boldsymbol{B}_{x}^{I I}$ and $\boldsymbol{B}_{z}^{I I}$ are obtained by:

$$
\begin{gathered}
\boldsymbol{B}_{x}^{I I}=-\mu_{0} j \frac{2 \pi}{\tau} \boldsymbol{N}_{x} \boldsymbol{\Phi}^{I I} \\
\boldsymbol{B}_{z}^{I I}=-\mu_{0} \frac{\partial \boldsymbol{\Phi}^{I I}}{\partial z}
\end{gathered}
$$

The transition from the matrix form to the spatial form is made using the relations:

$$
\begin{aligned}
& B_{x}^{I I}(x, y, z)=\left(\boldsymbol{B}_{x}^{I I}\right)^{T} e^{j\left(\frac{2 \pi \operatorname{diag}\left(\boldsymbol{N}_{x}\right) x}{\tau}+\frac{2 \pi \operatorname{diag}\left(\boldsymbol{N}_{\mathbf{y}}\right) \boldsymbol{y}}{L}\right)} \\
& B_{z}^{I I}(x, y, z)=\left(\boldsymbol{B}_{z}^{I I}\right)^{T} e^{j\left(\frac{2 \pi \operatorname{diag}\left(N_{x}\right) x}{\tau}+\frac{2 \pi \operatorname{diag}\left(\boldsymbol{N}_{\boldsymbol{y}}\right) \boldsymbol{y}}{L}\right)}
\end{aligned}
$$

Where the function $\operatorname{diag}(\boldsymbol{X})$ returns a column vector composed of the elements of the diagonal of the matrix $\boldsymbol{X}$ and $\boldsymbol{X}^{T}$ is the transpose of matrix $\boldsymbol{X}$.

Eventually, $\mathrm{x}$-component and the z-component of the flux modulation function are the ratio of $B_{x}^{I I}(x, y, z)$ and $B_{z}^{I I}(x, y, z)$ respectively and the magnetic flux density generated by the magnet without the bulk $B_{0}$ :

$$
\begin{aligned}
& F M_{x}^{I I}(x, y, z)=\frac{B_{x}^{I I}(x, y, z)}{B_{0}} \\
& F M_{z}^{I I}(x, y, z)=\frac{B_{z}^{I I}(x, y, z)}{B_{0}}
\end{aligned}
$$

Where $\mathrm{B}_{0}$ is obtained from the Ampere law:

$$
B_{0}=-\frac{B_{r e m}\left(Z_{3}-Z_{2}\right)}{Z_{3}}
$$

\section{Magnetic field of the whole inductor}

Once the flux modulation function is determined in the cartesian frame, the transition to the cylindrical frame is achieved by using the following relations between the coordinates:

$$
\begin{gathered}
r=R_{m}+y \\
\theta=\frac{x}{R_{m}}
\end{gathered}
$$

In the cylindrical frame, the z-component of the modulation function is identical to that of the cartesian frame $F M_{z}^{I I}(x, y, z)$ and the $\theta$-component is equal to the $\mathrm{x}$-component $F M_{x}^{I I}(x, y, z)$.

Ultimately, the magnetic flux density generated by the whole inductor is obtained from the following relations:

$$
\begin{aligned}
& B_{\text {rotorz }}(r, \theta, z)=B_{\text {coilz }}(r, z) \times F M_{z}^{I I}(x, y, z) \\
& B_{\text {rotor } \theta}(r, \theta, z)=B_{\text {coilz }}(r, z) \times F M_{x}^{I I}(x, y, z)
\end{aligned}
$$

Where $B_{\text {rotorz }}$ and $B_{\text {rotore }}$ are the z-component and the $\theta$-component respectively of the magnetic flux density produced by the inductor while $B_{\text {coilz }}$ is the magnetic flux density in the z-direction generated by the HTS-coil alone. $B_{\text {coilz }}$ is obtained using the 2-D axisymmetric model presented in [24].

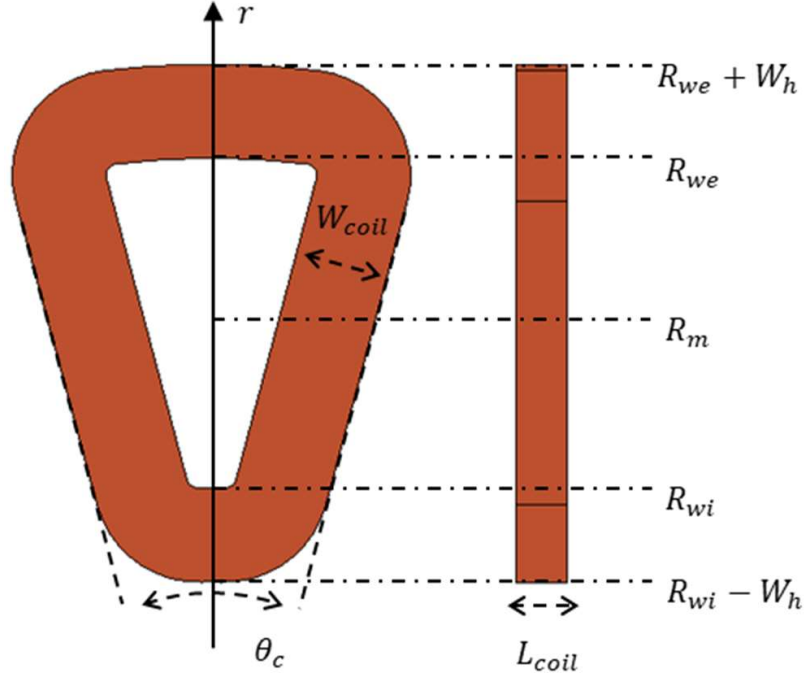

Fig. 7. Geometrical parameter of an armature coil.

\section{ELECTROMAGNETIC POWER AND EDDY-CURRENT LOSSES CALCULATION}

In a three-phase electrical machine, the electromagnetic torque $T_{e m}$ can be calculated from:

$$
T_{e m}=\frac{3 E_{1} I_{r m s}}{\Omega}
$$

Where $\Omega$ is the rotational speed, $\mathrm{I}_{\mathrm{rms}}$ is the rms value of the armature electrical current and $E_{1}$ is the rms value of the fundamental component of the electromotive force which is obtained from:

$$
E_{1}=\frac{p \Omega \lambda_{1}}{\sqrt{2}}
$$

Where $\lambda_{1}$ is the phase flux linkage amplitude due to the fundamental spatial harmonic of the inductor. In the present case, the air cored stator complexify the calculation of $\lambda_{1}$. Indeed, the inhomogeneity of the magnetic field in the armature region means that each turn of the windings has a different electromotive force. The expression used to account for the magnetic field inhomogeneities and calculate $\lambda_{l}$ is:

$$
\begin{aligned}
& \lambda_{1}=\frac{N_{s} K_{p} K_{d}}{L_{\text {coil }}} \times \\
& \int_{R_{w i}-W_{h}}^{R_{w e}+W_{h}} \int_{L_{\text {coil }} \frac{\pi}{p}} \int_{h} K_{h}(r) \widehat{B_{1}}(r, z) \cos (p \theta) r d r d \theta d z
\end{aligned}
$$

The geometric parameters involved in (76) are detailled in Fig. 7. $R_{w i}$ and $R_{w e}$ are respectively the inner and outer radius of the inner turn of a coil composing the armature. $W_{\text {coil }}$ is the width of a coil conductor bundle in the $\theta$-direction, $L_{\text {coil }}$ the thickness of a coil along the z-direction, $W_{h}$ the thickness of the coil head, $\theta_{c}$ the opening angle of a stator coil and $N_{s}$ the number of turns per phase. 


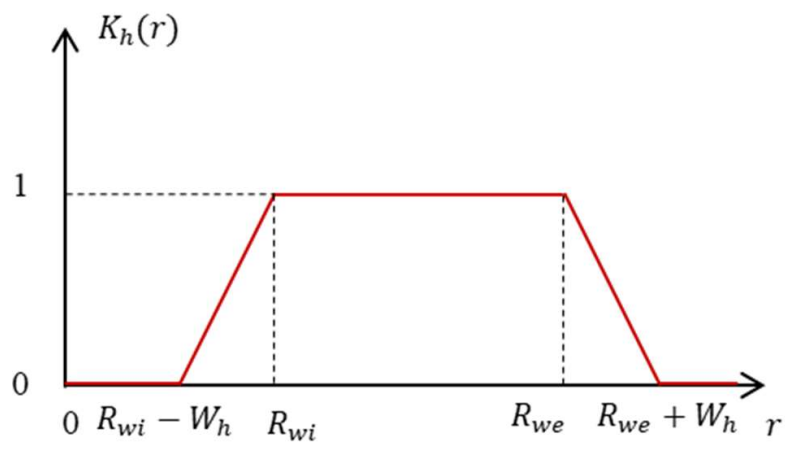

Fig. 8. Function $K_{h}(r)$ accounting for the coil head influence on the flux linkage.

Several kinds of windings can be modelled from (76) through the winding coefficients $K_{p}$ and $K_{d} . K_{d}$ is the distribution factor while $K_{p}$ is the pitch factor whose calculation is detailed for several types of axial flux air cored windings in [32]. In the following, only the case of a concentrated winding in which $W_{h}=W_{\text {coil }}$ will be detailed but conventional overlapping stator windings can be considered as well.

The value of $K_{d}$ can be classicaly calculated for differents windings according to [33]. In the case of concentrated windings, $K_{p}$ is expressed as:

$$
K_{p}=\frac{2}{p \theta_{m}} \sin \left(\frac{p}{2}\left(\theta_{c}-\theta_{m}\right)\right) \sin \left(\frac{p \theta_{m}}{2}\right)
$$

Where the angle $\theta_{m}$ is defined as:

$$
\theta_{m}=\frac{W_{\text {coil }}}{R_{m}}
$$

The fundamental spatial harmonic of the inductor $\widehat{B_{1}}(r, z)$ is obtained from the previous section inductor model as follows:

$$
\begin{aligned}
& \widehat{B_{1}}(r, z)=B_{\text {coilz }}(r, z) \times \\
& \left(\sum_{k=-N}^{N} \frac{\widehat{B_{z}^{I}}(z)}{B_{0}} e^{j \frac{2 \pi k}{L} y}+\frac{\widehat{B}_{-1, k}^{I}(z)}{B_{0}} e^{j \frac{2 \pi}{L} y}\right)
\end{aligned}
$$

The integrals over $r$ and $\theta$ in (76) allow the calculation of the flux under a pole. Since the flux value changes in the $\mathrm{z}$-direction, the third integral over $\mathrm{z}$ is made to calculate the average of this flux in the armature.

The function $K_{h}(r)$ accounts for the coil heads influence on the flux linkage. Indeed, the outermost region of the windings enclose more flux than the innermost region. Thus, $K_{h}(r)$ is represented in Fig. 8.

(a)

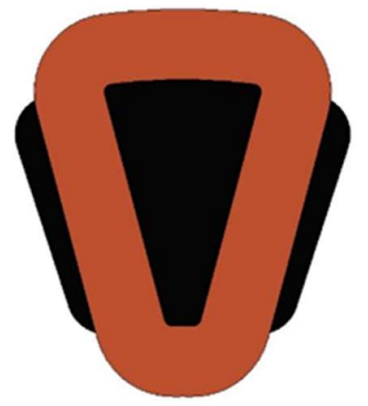

(b)

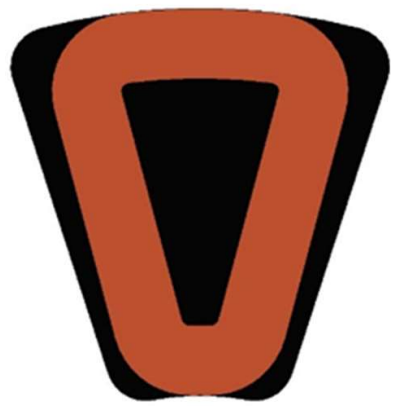

Fig. 9. Representation of the two main arrangements of the armature in relation to the bulks. (a) The bulks cover only the purely radial part of the armature giving the relations: $R_{e}=R_{w e}$ and $R_{i}=R_{w i}$. (b) The bulks also cover the coil heads giving the relations: $R_{e}=R_{w e}+W_{h}$ and $R_{i}=R_{w i}-$ $W_{h}$

The influence of the coil heads depends on the machine arrangement, of which the two main ones are shown in Fig. 9. On the one hand, in the case of Fig. 9 (a) where the coil heads are not covered by the bulks, their impact on the flux linkage is small and not considering $K_{h}(r)$ would not lead to a significant error on the torque. On the other hand, for Fig. 9 (b) where the coil heads are covered, omitting $K_{h}(r)$ could lead to an underestimation of the torque depending on the machine geometry. In any case, the computation of the triple integral of (76) is done numerically.

Another important consequence of the air cored armature topology is that the copper conductors are directly subjected to the magnetic field of the inductor. Hence, the eddy-current losses in the windings are higher than in iron cored armatures and the calculation of these losses is an important part of this machine sizing process.

The computation of the losses is done by neglecting the magnetic field generated by the armature currents and the associated losses as the main contribution to these losses comes from the inductor. The calculation is done for each turn by using the formula for the losses in a conductor subjected to an external magnetic field which can be found in [34]. The latter method for axial flux machines with an air cored armature is described in detail in [35].

(a)

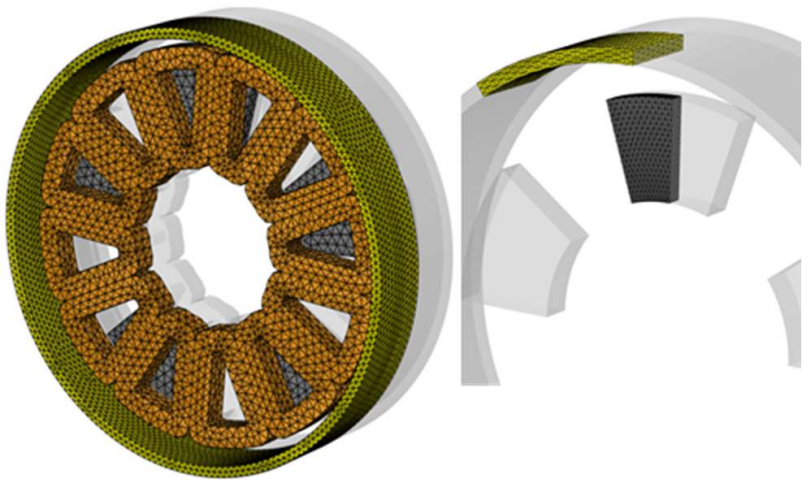

Fig. 10. Geometry and mesh of the CFEM (a) and SFEM (b). The grey regions are not simulated thanks to the symmetries. 


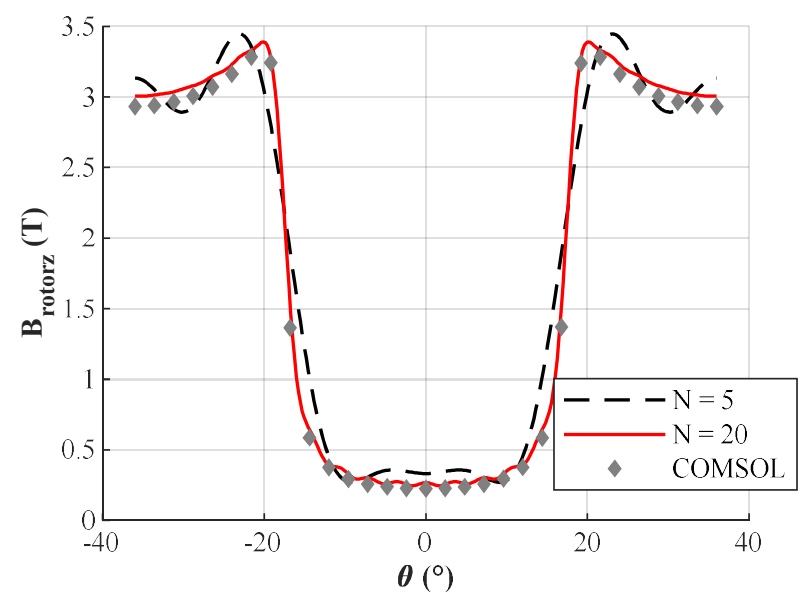

Fig. 11. Axial component of the inductor flux density at the average radius, $3 \mathrm{~mm}$ from the bulk surface $\left(r=R_{m}\right.$ and $\left.z=Z_{1}+3 \mathrm{~mm}\right)$.

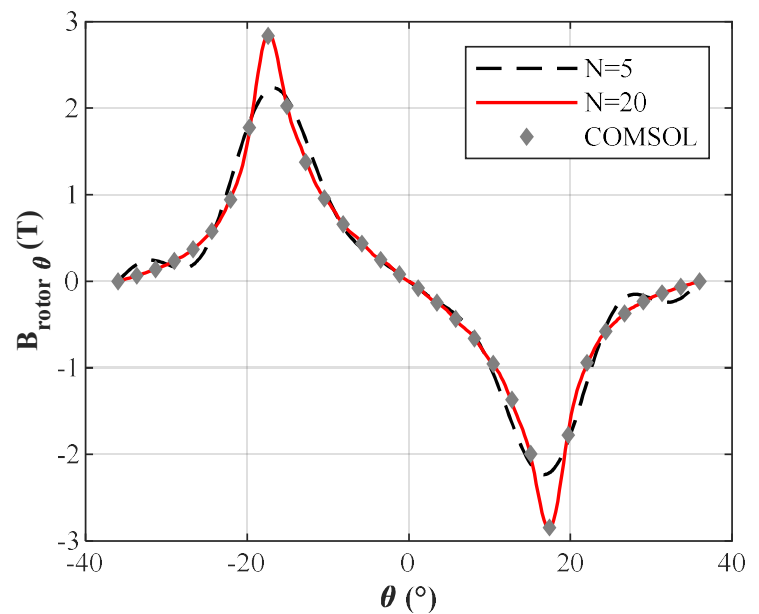

Fig. 12. Azimuthal component of the inductor flux density at the average radius, $3 \mathrm{~mm}$ from the bulk surface $\left(r=R_{m}\right.$ and $\left.z=Z_{1}+3 \mathrm{~mm}\right)$.

\section{COMPARISON WITH 3-D FEM SIMULATIONS}

In this section, the precision and efficiency of the proposed method will be assessed through the comparison of the three following models:

- The semi-analytical model (SAM) in which the inductor magnetic field is obtained from the model proposed in section 3 while the torque and the eddy current losses are calculated from the armature model of section 4. This model is implemented on the software MATLAB 2019.

- A semi-FEM model (SFEM) in which the inductor magnetic field is obtained from a 3-D stationary model in A-formulation with the software COMSOL Multiphysics while the torque and the eddy current losses are calculated from the armature model of section 4 .

- A complete FEM model (CFEM) in which the inductor and the armature are simulated on COMSOL with the torque being calculated using the Laplace force on the windings. The eddy current losses are not computed for this model.

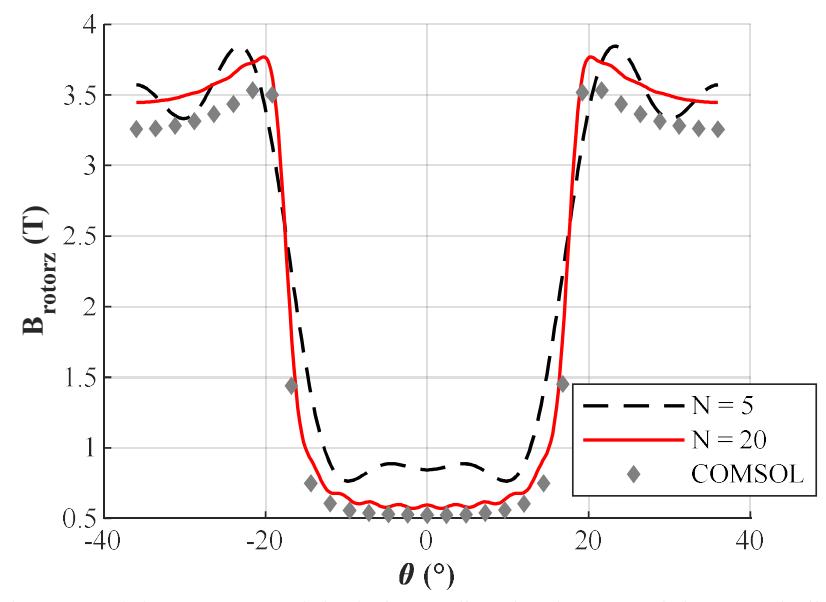

Fig. 13. Axial component of the inductor flux density around the outer bulk radius, $3 \mathrm{~mm}$ from the bulk surface $\left(r=R_{e}-10 \mathrm{~mm}\right.$ and $\left.z=Z_{1}+3 \mathrm{~mm}\right)$.

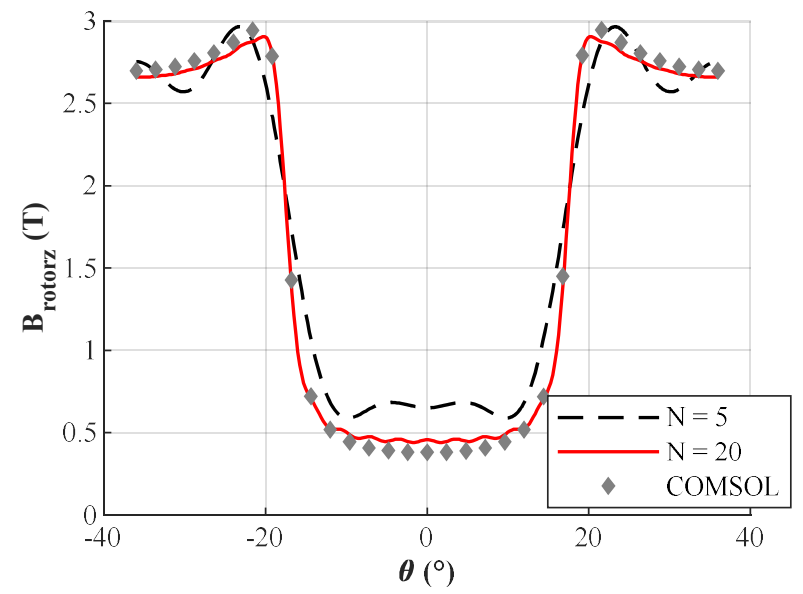

Fig. 14. Axial component of the inductor flux density around the inner bulk radius, $3 \mathrm{~mm}$ from the bulk surface $\left(r=R_{i}+10 \mathrm{~mm}\right.$ and $\left.z=Z_{1}+3 \mathrm{~mm}\right)$.

Fig. 10 shows the mesh and simulated geometry of the CFEM (a) and SFEM (b). As the winding layout represented is concentrated, the armature periodicity is different from the rotor periodicity. Therefore, the only symmetry plane is $(r \theta)$ in the CFEM whereas the SFEM model only need to consider $1 / 4$ of a bulk and the corresponding portion of the coil.

This study will identify the consequences of the different assumptions from which the SAM is derived. The different models will be tested with the parameters listed in Table. 1.

\section{A. Flux density distribution in the air gap}

Fig. 11 and Fig. 12 show the axial and azimuthal components of the flux density in the air gap at the mean radius obtained with the SAM for 5 and 20 harmonics and by the numerical models (SFEM and CFEM). For $N=20$, a good agreement is observed with the numerical simulations while $N=5$ also gives a reasonable estimate of the flux density distribution but does not allow predicting precisely the maximum value of the tangential component.

Fig. 13 and Fig. 14 show the axial component of the flux density in the air gap around the bulk outer and inner radii respectively. In both cases, the effects of the linearisation at the 
mean radius can be seen as the analytical curves slightly differ from the numerical curves. Nonetheless, the accuracy of the results seems to be more than satisfactory.

Additionally, Fig.15 shows the flux density norm in the winding region computed by the CFEM. It appears that the flux density is higher on the top portion of the armature compared to the lower part.

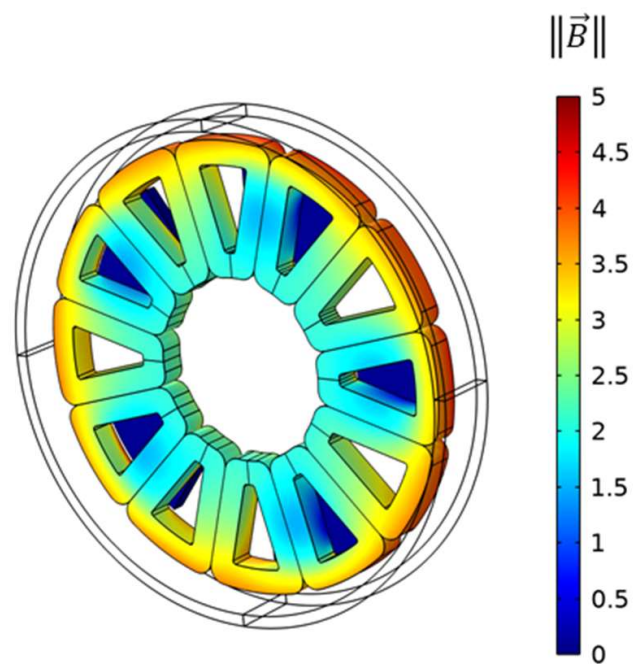

Fig. 15. Flux density norm distribution over the winding region from the CFEM model

TABLE. 1.

PARAMETERS OF THE STUDIED MACHINE.

\begin{tabular}{lll}
\hline \hline & & \\
Symbol & Description & Value \\
\hline$R_{e}$ & Bulk outer radius & $148 \mathrm{~mm}$ \\
$R_{i}$ & Bulk inner radius & $85 \mathrm{~mm}$ \\
$R_{m}$ & Bulk average radius & $116.5 \mathrm{~mm}$ \\
$R_{b}$ & HTS coil inner radius & $176 \mathrm{~mm}$ \\
$R_{w e}$ & Outer radius of the armature coil inner turn & $148 \mathrm{~mm}$ \\
$R_{w i}$ & Inner radius of the armature coil inner turn & $85 \mathrm{~mm}$ \\
$p$ & Number of pole pairs & 5 \\
$\alpha$ & Bulk pole-arc to pole-pitch ratio & 0.5 \\
$W_{c o i l}$ & Armature coil thickness & $18 \mathrm{~mm}$ \\
$L_{c o i l}$ & Armature coil length & $20 \mathrm{~mm}$ \\
$W_{b}$ & HTS coil thickness & $8.8 \mathrm{~mm}$ \\
$Z_{b}$ & HTS coil half length & $53.5 \mathrm{~mm}$ \\
$Z_{1}$ & Bulk half thickness & $10 \mathrm{~mm}$ \\
$E_{g}$ & Air-gap length & $3 \mathrm{~mm}$ \\
$d_{s}$ & Winding strand diameter & $0.25 \mathrm{~mm}$ \\
$K_{c u}$ & Winding filling factor & 0.7 \\
$N_{s} I_{r m s}$ & Ampere-turns per phase & $60.5 \mathrm{kA}$ \\
$J_{b}$ & HTS coil current density & $613 \mathrm{~A} / \mathrm{mm}{ }^{2}$ \\
$\Omega$ & Mechanical rotational speed & $5000 \mathrm{rpm}$ \\
\hline \hline
\end{tabular}

\section{B. Precision on the torque and the eddy current losses}

Observing the flux density distribution in the air gap is not sufficient to properly assess the model precision. For this purpose, the torque and the eddy current losses are more relevant values. Indeed, the torque indicates the overall accuracy of the fundamental over the whole armature area while the eddy current losses inform on the harmonic's precision.

First of all, Fig. 16 shows the computational time of the different models as a function of the number of harmonics.
Various elements can be observed. Firstly, the computation time of the SFEM increases a little with the number of harmonics while the CFEM time is constant. This is because the spectral analysis is only made for the eddy current losses computation in the SFEM while they are not calculated in the CFEM. In addition, the computation time of the SFEM is more important than CFEM because of this spectral analysis. Subsequently, the calculation time of the SAM rises exponentially with $N$ as it strongly increases the matrices sizes. However, the resolution of the SAM remains below the numerical methods for $N<20$ and is of the order of a second for $N<10$. Thus, as long as it is accurate enough for 10 harmonics, the proposed model is significantly faster than numerical methods.

In order to ascertain the latter condition for the torque, Fig. 17 shows the relative error in absolute value of the SAM compared to the SFEM and the CFEM as a function of $N$. The CFEM is here taken as a reference since it simulates the exact geometry of the inductor and armature. Thus, the comparison of the SAM and the CFEM provides the overall accuracy of the model while the comparison of the SAM and the SFEM only gives the inductor precision. The inductor model appears to be precise even for $N=5$ as the error between the SAM and the SFEM is below $4 \%$. Additionally, for $N=30$, the error between the SAM and the SFEM drops to $0.15 \%$. The overall accuracy of the model is also good as the maximum error is below $7 \%$. Eventually, it should be noted that the error between the SFEM and the CFEM is not influenced by $N$ as it corresponds to the armature model error and accounts for $3.2 \%$ of the overall error.

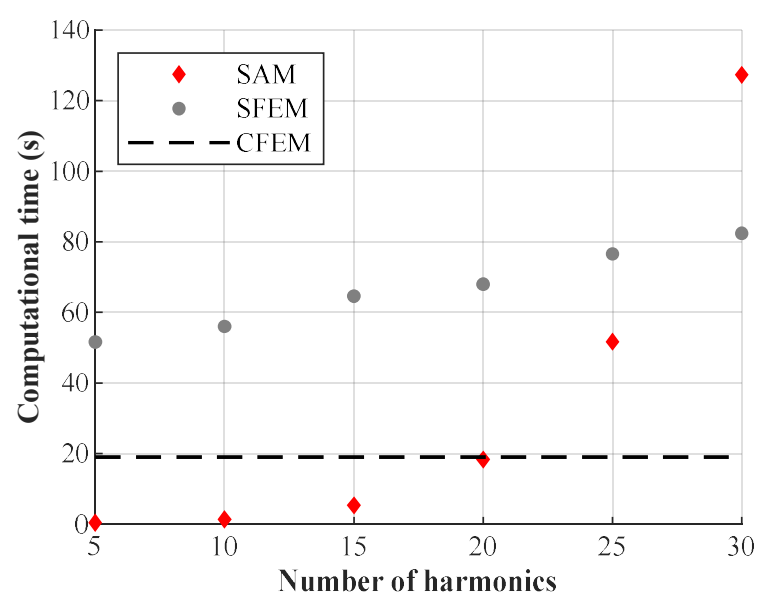

Fig. 16. Computational time of the different models versus the number of harmonics.

Moreover, Table. 2 compares the torque calculated by the different models for the machine of Table. 1 with and without the covering of the coil heads as presented on Fig. 9. This table illustrate the impact of the coil heads on the torque as was described in section 4 . When the coil heads are not covered, neglecting their impact, as done in the SFEM (b), lead to an additional $4 \%$ error. In contrast, when the coil heads are covered, the additional error made by the SFEM (b) is $15.5 \%$. 
This table also shows the significant performance improvement that can be achieved by covering the coil heads.

TABLE. 2. TORQUE CALCULATED BY EACH MODEL FOR THE CASES WHERE THE COIL HEADS ARE COVERED AND UNCOVERED. (A) NORMAL SFEM. (B) SFEM MODEL WITH THE FLUX LINKAGE COMPUTED ONLY ON THE PURELY RADIAL PART OF THE WINDINGS.

\begin{tabular}{lll}
\hline \hline & & \\
Model & Coil heads uncovered & Coil heads covered \\
\hline CFEM & $1366 \mathrm{Nm}$ & $2206 \mathrm{Nm}$ \\
SAM N $=10$ & $1301 \mathrm{Nm}$ & $2050 \mathrm{Nm}$ \\
SFEM (a) & $1322 \mathrm{Nm}$ & $2114 \mathrm{Nm}$ \\
SFEM (b) & $1254 \mathrm{Nm}$ & $1711 \mathrm{Nm}$ \\
\hline
\end{tabular}

Eventually, Fig. 18 presents the normalized eddy current losses obtained by the SAM and the SFEM as a function of the number of harmonics. For this analysis, the reference value is represented by the losses computed by the SFEM with $N=30$ as it considers the exact magnetic field with a large number of harmonics. The first thing to observe here is the inaccuracy of the SAM for $N=5$. Indeed, the value calculated with the SAM is $27 \%$ lower than the reference value but the spread decreases rapidly as the number of harmonics increase. Hence, while 5 harmonics are sufficient to get an accurate idea of the torque, taking less than 10 harmonics leads to a significant error on the eddy current losses.

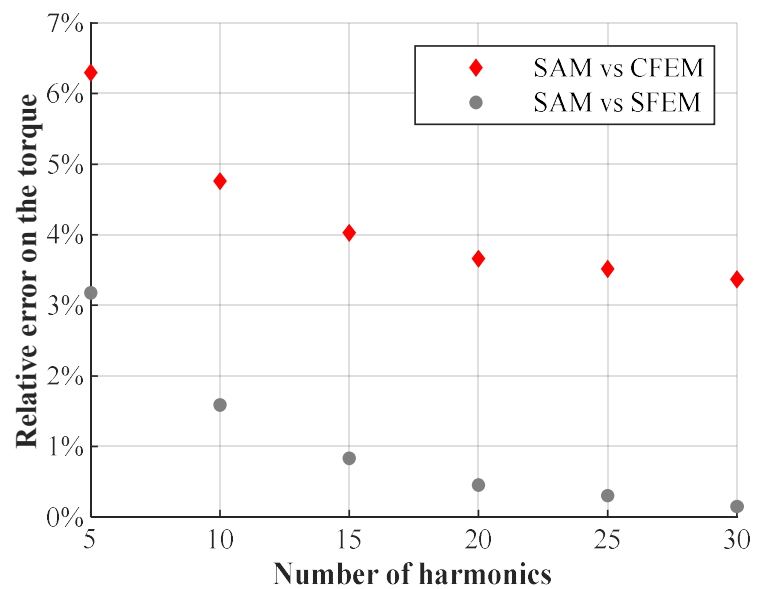

Fig. 17. Relative error in absolute value on the torque of the SAM compared to the SFEM and the CFEM.

Nevertheless, it is important to distinguish between the two sources of error here. Firstly, the eddy current losses calculation requires a sufficient number of harmonics since high ranks harmonics still generate losses that are not negligible. This source of error is present even if the magnetic field distribution is completely accurate and corresponds to the error made by the SFEM. Thus, above $\mathrm{N}=20$, this error is almost null while it accounts for $18.4 \%$ of the total error at $N=5$. Subsequently, the other source of error is the inaccuracy of the flux density distribution obtained from the SAM. As all harmonics are interdependent, a large number of harmonics is required to calculate their value accurately. It can therefore be deduced that this source of error is responsible for $8.6 \%$ of the total error at $N=5$.

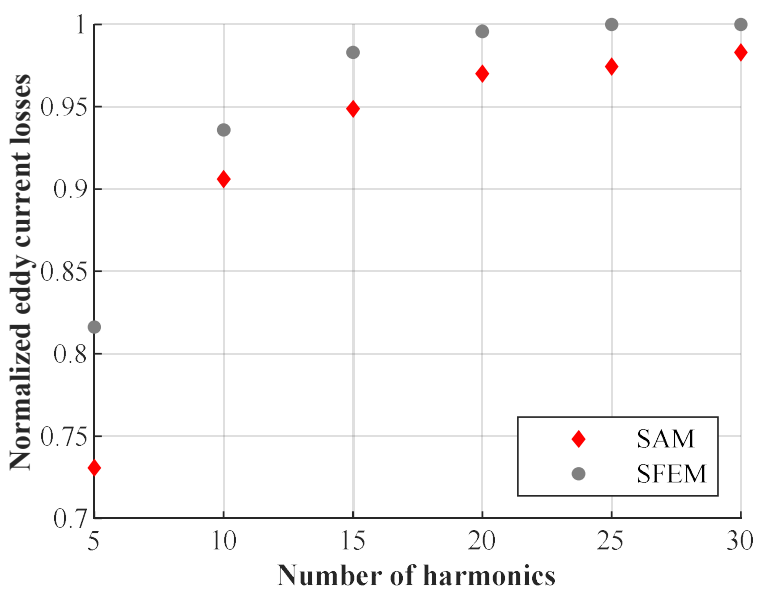

Fig. 18. Normalized eddy current losses calculated by the SAM and the SFEM versus the number of harmonics.

\section{Influence of the geometrical parameters}

According to the previous results, the SAM with $N=10$ would appear to be a good compromise between accuracy and calculation speed. However, the geometrical parameters presented in Table. 1 are the result of an optimisation from previous works using the SFEM model. Therefore, it is important to investigate the sensitivity of the model's accuracy with the variation of the geometric parameters. For this purpose, the following parameters will be explored:

- $R_{w e}$ in the range [100 $\mathrm{mm}: 250 \mathrm{~mm}$.

- The ratio $R_{w e} / R_{w i}$ in the range [0.45:0.7].

- $\quad \alpha$ in the range $[0.3: 0.7]$.

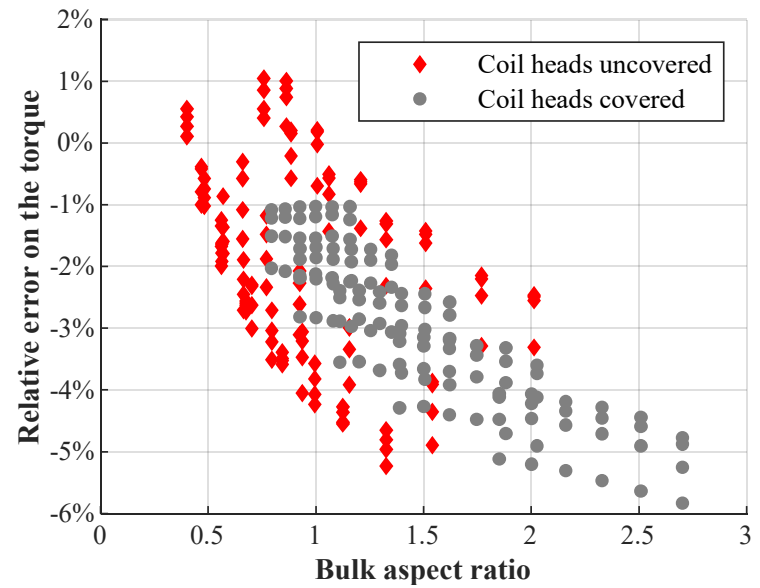

Fig. 19. Relative error on the torque of the SAM with $N=10$ compared to the SFEM for the explored parameters.

Additionally, the study will be performed for the cases represented in Fig. 9 (a) and Fig. 9 (b) with the coil heads covered or not by the bulks. Within this context, the aspect ratio of the bulk is defined as follows:

$$
\text { Aspect ratio }=\frac{R_{e}-R_{i}}{\alpha \tau}
$$

Since all the parameters explored affect the aspect ratio, the latter seems to be a relevant value to understand the results. 
Fig. 19 shows the relative error on the torque of the SAM with $N=10$ compared to the SFEM function of the aspect ratio. Several elements can be observed. Firstly, there is a clear correlation between the relative error and the aspect ratio as linearisation at the mean radius has less impact on bulks with low aspect ratios. For the same reason, the model is less precise for machines with coil heads covered. Secondly, it can be observed that the SAM has a strong tendency to underestimate the torque. Lastly, the SAM accuracy remains good even for large aspect ratios as the maximum error is inferior to $6 \%$. It should also be noted that, as for today, the use of bulks with very small or very large aspect ratios is unlikely since ring segment shaped bulks are mostly manufactured by machining disk or square shaped bulks [36]. Thus, HTS bulks with an aspect ratio close to 1 are easier to produce.

Fig. 20 shows the relative error on the eddy current losses of the SAM with $N=10$ compared to SFEM function of the aspect ratio. The same observations as in Fig. 19 can be made. Indeed, there is a correlation between the error and the aspect ratio and the SAM tends to underestimate the losses. However, it is clear that the aspect ratio is not the only parameter influencing the error. Additionally, the average error is much higher for the losses than for the torque as was observed for the parameters of Table. 1. Indeed, as previously said, every harmonics of the SAM model are interdependent, which means that to obtain an accurate value of 10 harmonics, many more must be calculated.

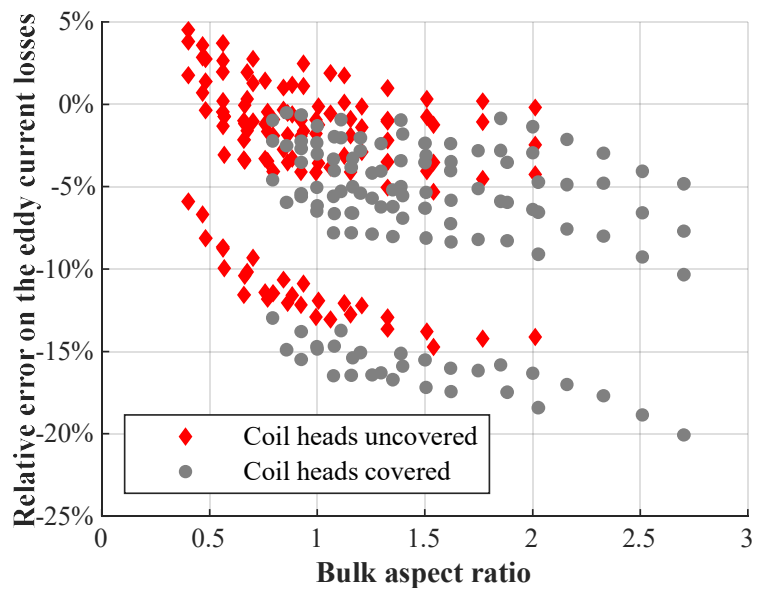

Fig. 20. Relative error on the eddy current losses of the SAM with $N=10$ compared to the SFEM for the explored parameters.

\section{FLUX PENETRATION IN THE BULK}

The method described above as well as the numerical simulation are based on the main assumption that the bulk are perfectly diamagnetic which corresponds to type II superconductors with an infinite critical current density. However, even the most efficient superconductors does not have a perfect diamagnetic behaviour [36]. Therefore, it is important for the model to approach the bulk magnetic behaviour in order to have a more accurate idea of the performance of a flux modulation machine. The most common method to finely simulate this behaviour would be a time- dependant H-formulation considering the E-J law [37]-[39]. However, the computation in 3-D takes several minutes making it difficult to integrate into a sizing study. Alternatively, the SAM can be improved using the Bean model [40] to account for the magnetic penetration in the bulk.

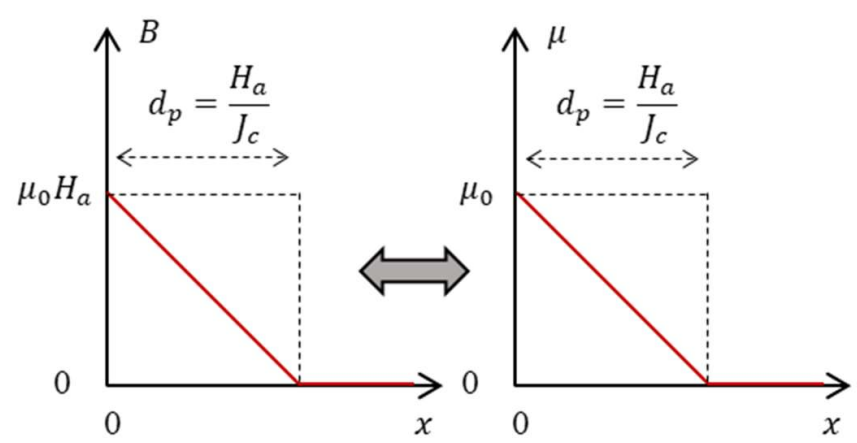

Fig. 2I. Implementation principle of the Bean model in the SAM modeI. The penetration of the magnetic field is modelled as a variation of the permeability instead of a current density.

Fig. 21 presents the implementation principle of the Bean model in the SAM model. The Bean model considers a linear penetration of the magnetic field at the edges of the superconductor. The penetration depth of the magnetic field $d_{p}$ is linked to the bulk critical current $J_{c}$ and the applied magnetic field $H_{a}$ through the following relation:

$$
d_{p}=\frac{H_{a}}{J_{c}}
$$

In the SAM, complex permeability distributions can be considered. Hence, the penetration of the magnetic field is considered by a linear decline of the permeability with a penetration depth calculated from (81). The value of $H_{a}$ is computed using the coil axisymmetric model [24] giving the following relation:

$$
H_{a}=\frac{B_{\text {coilz }}\left(R_{m}, 0\right)}{\mu_{0}}
$$

Thus, to include this feature in the SAM, only the matrix $\boldsymbol{\mu}_{c}^{I}$ has to be changed. To do so, the Fourier series coefficients $\hat{\mu}_{n, k}$ of the permeability need to be recalculated. Fig. 22 shows the permeability distribution of the region I for an incomplete penetration of the bulk. Since the permeability is symmetric along $\mathrm{x}$ and $\mathrm{y}$, only a quarter of the region $\mathrm{I}$ needs to be considered for the Fourier series coefficients calculation. The bulk centre is still diamagnetic but the permeability in the three sectors at the edges is expressed as:

In sector (a):

$$
\mu^{I}(x, y)=\frac{\mu_{0}}{d_{p}}\left(y-\frac{D}{2}+d_{p}\right)
$$

In sector (b):

$$
\mu^{I}(x, y)=\frac{\mu_{0}}{d_{p}}\left(x-\frac{\alpha \tau}{2}+d_{p}\right)
$$

In sector $(c)$ :

$$
\mu^{I}(x, y)=\mu_{0}\left(1-\frac{\left(x-\frac{\alpha \tau}{2}\right)\left(y-\frac{D}{2}\right)}{d_{p}^{2}}\right)
$$


The coefficients $\hat{\mu}_{n, k}$ are then calculated from:

$$
\begin{aligned}
& \hat{\mu}_{n, k}= \\
& \frac{4}{\tau L} \int_{0}^{\tau} \int_{0}^{L} \mu^{I}(x, y) \cos \left(2 \pi \frac{n x}{\tau}\right) \cos \left(2 \pi \frac{k y}{L}\right) d x d y
\end{aligned}
$$

Care should be taken as Fig. 22 represents only the incomplete penetration case. Indeed, three other cases should be considered:

The penetration is complete in the y-direction only $\left(d_{p}>\frac{D}{2}\right.$ and $d_{p}<\frac{\alpha \tau}{2}$ ) and only sectors (a) and (c) are present in the bulk.

The penetration is complete in the $\mathrm{x}$-direction only $\left(d_{p}<\frac{D}{2}\right.$ and $d_{p}>\frac{\alpha \tau}{2}$ ) and only sectors (b) and (c) are present in the bulk.

The penetration is complete in both directions $\left(d_{p}>\frac{D}{2}\right.$ and $d_{p}>\frac{\alpha \tau}{2}$ ) and the sector (c) recovers the whole bulk.

Once again, $10^{-4} \mu_{0}$ must be added to the $\hat{\mu}_{0,0}$ coefficient such that the matrix $\boldsymbol{\mu}_{c}^{I}$ is invertible.

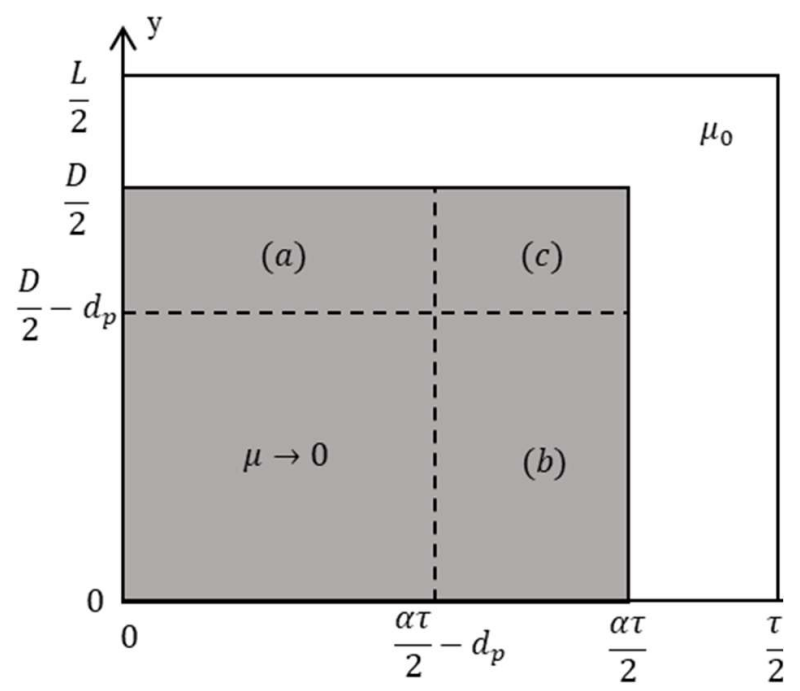

Fig. 22. Magnetic permeability distribution in a quarter of the region I for an incomplete penetration.

In order to evaluate the relevancy of the improved SAM (ISAM), another reference model is made on COMSOL (HFEM). This model is a 3-D time dependant simulation of the inductor using an $\mathrm{H}$-formulation of the Maxwell equations. The superconducting bulk is modelled through an E-J law with a constant $J_{c}$ [38] and the power law index equal to 20 . The coil is supplied with a current ramp up to its nominal value in $60 \mathrm{~s}$ and then the current is kept constant for $60 \mathrm{~s}$ to reach the steady state at which the torque is computed using the armature model of section 4 .

Fig. 23 shows the current density distribution normalized to $J_{c}$ in the bulk calculated by the HFEM. It should be noted that the symmetries exploited here are identical as in the SFEM, only $1 / 4$ is therefore simulated. (a)

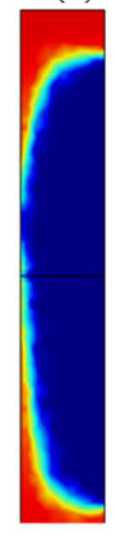

(b)

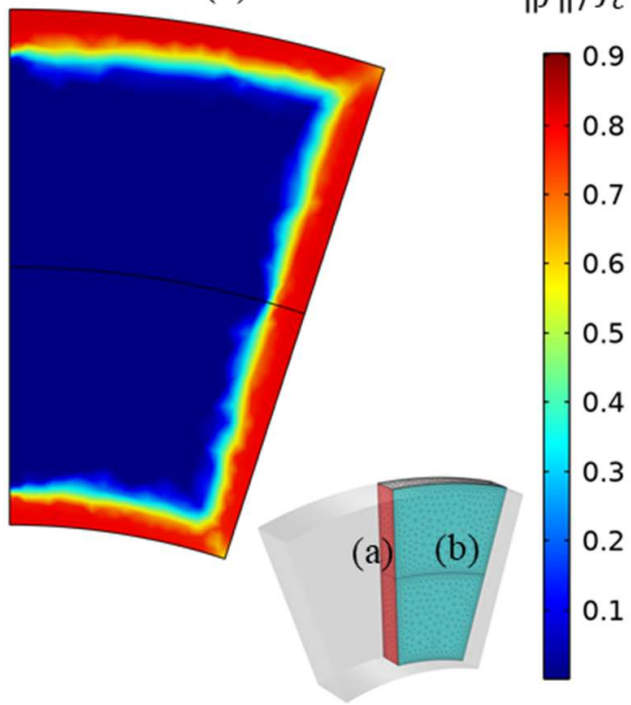

Fig. 23. Normalized current distribution in $1 / 4$ the bulk at its centre calculated by the HFEM for $J_{c}=1000 \mathrm{~A} / \mathrm{mm}^{2}$ : (a) corresponds to the $(\mathrm{rz})$ cross section highlighted in red; (b) corresponds to the $(r \theta)$ cross section highlighted in cyan.

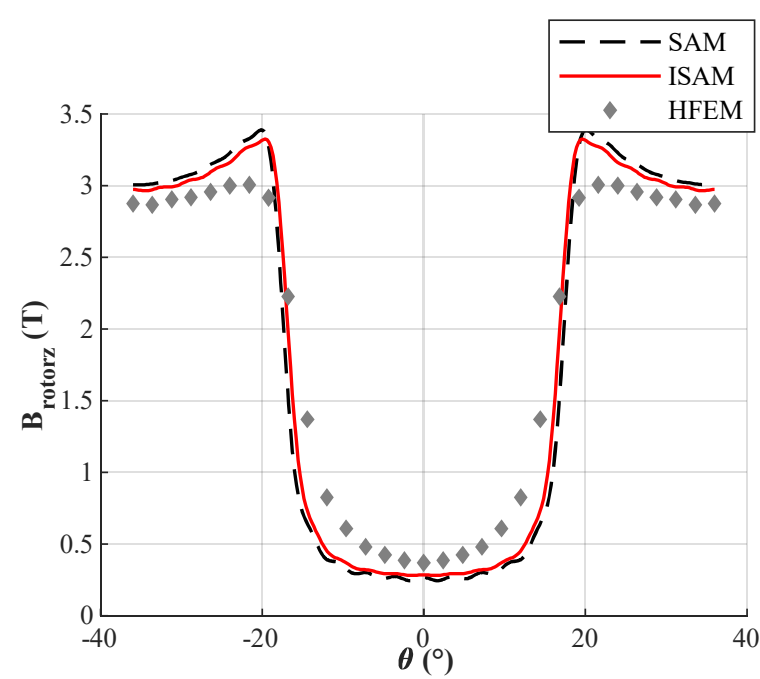

Fig. 24. Axial component of the inductor flux density at the average radius, $3 \mathrm{~mm}$ from the bulk surface $\left(r=R_{m}\right.$ and $\left.z=Z_{1}+3 \mathrm{~mm}\right)$ for $J_{c}=1000 \mathrm{~A} / \mathrm{mm}^{2}$ and $\mathrm{N}=20$.

Fig. 24 shows the axial component of the flux density in the air gap obtained with the SAM and ISAM for $\mathrm{N}=20$ compared to the HFEM for $J_{c}=1000 \mathrm{~A} / \mathrm{mm}^{2}$. It can be observed that the HTS bulk behaviour as predicted by the HFEM is not completely diamagnetic for this $J_{c}$. The modulation predicted by the ISAM differs from that of a diamagnetic bulk, though there is still a difference between the penetration calculated by the HFEM and the ISAM.

Fig. 24 is not sufficient to properly assess the relevancy of the ISAM as the behaviour still seems to be closer to perfect diamagnetism than to the actual behaviour as calculated by the HFEM. For this purpose, Fig. 25 shows the electromagnetic torque calculated by the HFEM and the ISAM for $\mathrm{N}=10$ 
function of the bulk critical current density. The torque value is normalized to the diamagnetic torque as the latter represents the maximum achievable torque. In both cases, a transition is observed from complete penetration at low $J_{c}$ to close to diamagnetism behaviour at high $J_{c}$ and both models predict similar behaviours. Yet, the transition does not occur at the same $J_{c}$ values and there is an important difference between $100 \mathrm{~A} / \mathrm{mm}^{2}$ and $1000 \mathrm{~A} / \mathrm{mm}^{2}$ which leads to an important error on the torque. Above the latter threshold, both curves are converging to the diamagnetic behaviour. Thus, the application of the Bean model in the ISAM allows to predict the global trend in magnetic behavior but cannot accurately calculate the torque for bulk with a small $J_{c}$. This can be explained by the geometrical assumptions of the Bean model which can be applied for infinitely long bulks. In the present case, the bulk thickness in the z-direction is the smallest dimension of the bulk, hence the end effects are very important and cannot be accounted for as the convolution method only allows the permeability to be variable in the $\mathrm{x}$ and $\mathrm{y}$ directions.

Indeed, as shown on Fig.23, altough the current density is located at the bulk edges as predicted by the Bean model, the latter predict that its value is either $J_{c}$ or 0 which is not exactly the case. Additionnaly, the currents also penetrate the bulk through the top and bottom surfaces of the bulk (i.e. surfaces along the $(r \theta)$ plane) which is not taken into account in the HFEM.

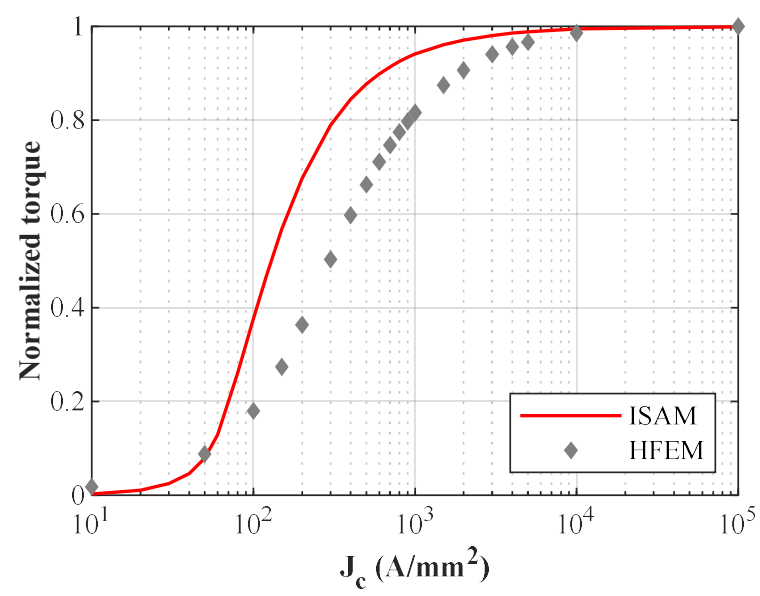

Fig. 25. Electromagnetic torque versus the bulk critical current density for the ISAM $(\mathrm{N}=10)$ and the HFEM. The torque value is normalized to the diamagnetic torque.

Nevertheless, the ISAM model is still more accurate than the SAM model and approaches the HFEM results for high $J_{c}$. It should be noted that the materials most likely to be used for magnetic shielding are $\mathrm{RE}-\mathrm{Ba}-\mathrm{Cu}-\mathrm{O}$ which have $J_{c}$ above $3000 \mathrm{~A} / \mathrm{mm}^{2}$ at temperatures close to liquid hydrogen [25] which places them within the area of validity of the ISAM. Lastly, the ISAM requires around a second of computation time for $N=10$ while the HFEM requires between 15 minutes and 3 hours to be solved depending on the $J_{c}$ value which makes it impossible to be integrated in an optimisation routine. Moreover, this high computationnal cost make is difficult to use a thin mesh which explain the rough aspect of the current density map of Fig.23.

Therefore, the ISAM model appears to be the best compromise between precision and calculation time but must be used carefully as it is suitable only for bulks with a behaviour close to the diamagnetism.

\section{CONCLUSION}

In this paper, a 3-D semi-analytical model of a superconducting axial flux modulation machine has been developed to calculate the torque and the eddy-current losses. The inductor model has been made using separation of variables method, Fourier series analysis and the application of the convolution theorem. This last tool allowed to consider domains with a variable permeability in two directions and enabled a resolution in 3-D using only 3 domains and therefore a drastically reduced computation time. The torque was derived from the electromotive force whereas the eddy-current losses were calculated from well known analytical formulas and the inductor flux density distribution. Eventually, as the variable permeability domains permit considering complex permeability distribution, the model has been improved using the Bean model to consider the magnetic penetration in the HTS bulk.

Comparisons with the FEM simulations have shown the ability of the proposed model to quickly provide a precise result when the bulks are considered diamagnetic. Indeed, for 10 harmonics, the torque and the eddy-current losses of the test machine can be predicted with less than $5 \%$ error for the torque and $10 \%$ error for the losses in less than 2 seconds. When the critical current density of the bulk is considered, the model has shown its ability to predict the trend in magnetic behaviour but does not accurately simulate bulks with low current densities. Thus, the validity area of this model still only comprises bulks with high critical current density and appears to be the best compromise between computation time and precision. In any case, the performances of the magnetic screens is the cornerstone of high specific power flux modulation machines. Thus, for aicraft applications, only large HTS bulks with large and homogeneous critical current density are relevant and likely to be used.

The model presented in this article is a powerful tool for optimisation and sizing processes. It allows efficient design for prototypes as well as extrapolations and theoretical studies on the potential of this machine technology for aeronautical applications. Additionnaly, the modelling method in 3-D can be adapted to other kind of axial flux machines with region with a variable permeability such as axial flux permanent magnet machines or switch reluctance couplings.

\section{ACKNOWLEDGEMENTS}

The authors would like to thank the Direction Générale de l'Armement (DGA), the Agence de l'Innovation de Défense (AID), and the Agence Nationale de la Recherche (ANR). This work was supported by the project FROST under Grant ANR19-ASMA-0001k. 


\section{REFERENCES}

[1] M. Boll et al., 'A holistic system approach for short range passenger aircraft with cryogenic propulsion system', Supercond. Sci. Technol., vol. 33, no. 4, p. 044014, Mar. 2020, doi: 10.1088/1361-6668/ab7779.

[2] K. S. Haran et al., 'High power density superconducting rotating machines - development status and technology roadmap', Supercond. Sci. Technol., vol. 30, no. 12, p. 123002, Nov. 2017, doi: 10.1088/13616668/aa833e.

[3] F. Berg, J. Palmer, P. Miller, M. Husband, and G. Dodds, 'HTS Electrical System for a Distributed Propulsion Aircraft', IEEE Transactions on Applied Superconductivity, vol. 25, no. 3, pp. 1-5, Jun. 2015, doi: 10.1109/TASC.2014.2384731.

[4] N. A. of S. Medicine Engineering, and, D. on E. and P. Sciences, A. and S. E. Board, and C. on P. and E. S. to R. C. A. C. Emissions, Commercial Aircraft Propulsion and Energy Systems Research: Reducing Global Carbon Emissions. National Academies Press, 2016.

[5] M. Filipenko et al., 'Concept design of a high power superconducting generator for future hybrid-electric aircraft', Supercond. Sci. Technol., vol. 33, no. 5, p. 054002, Mar. 2020, doi: 10.1088/1361-6668/ab695a.

[6] M. Corduan, M. Boll, R. Bause, M. P. Oomen, M. Filipenko, and M. Noe, 'Topology Comparison of Superconducting AC Machines for Hybrid Electric Aircraft', IEEE Transactions on Applied Superconductivity, vol. 30, no. 2, pp. 1-10, Mar. 2020, doi: 10.1109/TASC.2019.2963396.

[7] M. Corduan, 'Design of superconducting AC machines for hybridelectric aircraft', Karlsruher Institut für Technologie (KIT), 2021. [Online]. https://publikationen.bibliothek.kit.edu/1000129218

[8] P. J. Masson, J. E. Pienkos, and C. A. Luongo, 'Scaling Up of HTS Motor Based on Trapped Flux and Flux Concentration for Large Aircraft Propulsion', IEEE Transactions on Applied Superconductivity, vol. 17, no. 2, pp. 1579-1582, Jun. 2007, doi: 10.1109/TASC.2007.898111.

[9] P. J. Masson and C. A. Luongo, 'High power density superconducting motor for all-electric aircraft propulsion', IEEE Transactions on Applied Superconductivity, vol. 15, no. 2, pp. 2226-2229, Jun. 2005, doi: 10.1109/TASC.2005.849618.

[10] G. Malé, T. Lubin, S. Mezani, and J. Lévêque, 'Analytical calculation of the flux density distribution in a superconducting reluctance machine with HTS bulks rotor', Mathematics and Computers in Simulation, vol. 90, pp. 230-243, Apr. 2013, doi: 10.1016/j.matcom.2013.01.003.

[11] P. J. Masson, M. Breschi, P. Tixador, and C. A. Luongo, 'Design of HTS Axial Flux Motor for Aircraft Propulsion', IEEE Transactions on Applied Superconductivity, vol. 17, no. 2, pp. 1533-1536, Jun. 2007 doi: 10.1109/TASC.2007.898120.

[12] A. Colle, T. Lubin, and J. Leveque, 'Design of a superconducting machine and its cooling system for an aeronautics application', Eur Phys. J. Appl. Phys., vol. 93, no. 3, Art. no. 3, Mar. 2021, doi: $10.1051 /$ epjap/2020200027.

[13] A. Colle, T. Lubin, S. Ayat, O. Gosselin, and J. Leveque, 'Test of a Flux Modulation Superconducting Machine for Aircraft', J. Phys.: Conf. Ser., vol. 1590 , p. 012052, Jul. 2020, doi: 10.1088/17426596/1590/1/012052.

[14] E. Devillers, J. L. Besnerais, T. Lubin, M. Hecquet, and J. Lecointe, 'A review of subdomain modeling techniques in electrical machines: Performances and applications', in 2016 XXII International Conference on Electrical Machines (ICEM), Sep. 2016, pp. 86-92. doi: 10.1109/ICELMACH.2016.7732510.

[15] T. Lubin, S. Mezani, and A. Rezzoug, '2-D Exact Analytical Model for Surface-Mounted Permanent-Magnet Motors With Semi-Closed Slots', IEEE Transactions on Magnetics, vol. 47, no. 2, pp. 479-492, Feb. 2011, doi: 10.1109/TMAG.2010.2095874.

[16] E. Devillers, J. L. Besnerais, T. Lubin, M. Hecquet, and J. Lecointe, 'An Improved 2-D Subdomain Model of Squirrel-Cage Induction Machine Including Winding and Slotting Harmonics at Steady State', IEEE Transactions on Magnetics, vol. 54, no. 2, pp. 1-12, Feb. 2018, doi: 10.1109/TMAG.2017.2782222.

[17] T. Lubin, S. Mezani, and A. Rezzoug, 'Exact Analytical Method for Magnetic Field Computation in the Air Gap of Cylindrical Electrical Machines Considering Slotting Effects', IEEE Transactions on Magnetics, vol. 46, no. 4, pp. 1092-1099, Apr. 2010, doi: 10.1109/TMAG.2009.2036257.

[18] T. Lubin, S. Mezani, and A. Rezzoug, 'Improved Analytical Model for Surface-Mounted PM Motors Considering Slotting Effects and
Armature Reaction', Progress In Electromagnetics Research, vol. 25, pp. 293-314, 2010, doi: 10.2528/PIERB10081209.

[19] K. J. Meessen, B. L. J. Gysen, J. J. H. Paulides, and E. A. Lomonova, 'General Formulation of Fringing Fields in 3-D Cylindrical Structures Using Fourier Analysis', IEEE Transactions on Magnetics, vol. 48, no. 8, pp. 2307-2323, Aug. 2012, doi: 10.1109/TMAG.2012.2192939.

[20] T. Lubin, S. Mezani, and A. Rezzoug, 'Development of a 2-D Analytical Model for the Electromagnetic Computation of Axial-Field Magnetic Gears', IEEE Transactions on Magnetics, vol. 49, no. 11, pp. 5507-5521, Nov. 2013, doi: 10.1109/TMAG.2013.2267746.

[21] T. Lubin and A. Rezzoug, 'Improved 3-D Analytical Model for AxialFlux Eddy-Current Couplings With Curvature Effects', IEEE Transactions on Magnetics, vol. 53, no. 9, pp. 1-9, Sep. 2017, doi: 10.1109/TMAG.2017.2714628.

[22] B. Dolisy, S. Mezani, T. Lubin, and J. Lévêque, 'A New Analytical Torque Formula for Axial Field Permanent Magnets Coupling', IEEE Transactions on Energy Conversion, vol. 30, no. 3, pp. 892-899, Sep. 2015, doi: 10.1109/TEC.2015.2424159.

[23] T. Lubin and A. Rezzoug, '3-D Analytical Model for Axial-Flux EddyCurrent Couplings and Brakes Under Steady-State Conditions', IEEE Transactions on Magnetics, vol. 51, no. 10, pp. 1-12, Oct. 2015, doi: 10.1109/TMAG.2015.2455955.

[24] A. Colle, T. Lubin, S. Ayat, O. Gosselin, and J. Lévêque, 'Analytical Model for the Magnetic Field Distribution in a Flux Modulation Superconducting Machine', IEEE Transactions on Magnetics, vol. 55, no. 12, pp. 1-9, Dec. 2019, doi: 10.1109/TMAG.2019.2935696.

[25] V. Antal et al., 'Relationship between local microstructure and superconducting properties of commercial YBa2Cu3O7-\$lupdelta\$ bulk', Supercond. Sci. Technol., vol. 33, no. 4, p. 044004, Feb. 2020, doi: 10.1088/1361-6668/ab714f.

[26] Z. Djelloul-Khedda, K. Boughrara, F. Dubas, A. Kechroud, and B. Souleyman, 'Semi-Analytical Magnetic Field Predicting in Many Structures of Permanent-Magnet Synchronous Machines Considering the Iron Permeability', IEEE Transactions on Magnetics, vol. 54, no. 7 , pp. 1-21, Jul. 2018, doi: 10.1109/TMAG.2018.2824278.

[27] Z. Djelloul-Khedda, K. Boughrara, F. Dubas, and R. Ibtiouen, 'Nonlinear Analytical Prediction of Magnetic Field and Electromagnetic Performances in Switched Reluctance Machines', IEEE Transactions on Magnetics, vol. 53, no. 7, pp. 1-11, Jul. 2017, doi: 10.1109/TMAG.2017.2679686.

[28] R. L. J. Sprangers, J. J. H. Paulides, B. L. J. Gysen, and E. A. Lomonova, 'Magnetic Saturation in Semi-Analytical Harmonic Modeling for Electric Machine Analysis', IEEE Transactions on Magnetics, vol. 52, no. 2, pp. 1-10, Feb. 2016, doi: 10.1109/TMAG.2015.2480708

[29] H. Zhao, C. Liu, Z. Song, and J. Yu, 'Analytical Modeling and Comparison of Two Consequent-Pole Magnetic-Geared Machines for Hybrid Electric Vehicles', Energies, vol. 12, no. 10, Art. no. 10, Jan. 2019, doi: 10.3390/en12101888.

[30] H. S. Zhang, Z. X. Deng, M. L. Yang, Y. Zhang, J. Y. Tuo, and J. Xu, 'Analytical Prediction of Halbach Array Permanent Magnet Machines Considering Finite Tooth Permeability', IEEE Transactions on Magnetics, vol. 56, no. 6, pp. 1-10, Jun. 2020, doi: 10.1109/TMAG.2020.2982844

[31] O. De La Barriere, S. Hlioui, H. Ben Ahmed, M. Gabsi, and M. LoBue, '3-D Formal Resolution of Maxwell Equations for the Computation of the No-Load Flux in an Axial Flux Permanent-Magnet Synchronous Machine', IEEE Transactions on Magnetics, vol. 48, no. 1, pp. 128136, Jan. 2012, doi: 10.1109/TMAG.2011.2167347.

[32] M. J. Kamper, R.-J. Wang, and F. G. Rossouw, 'Analysis and Performance of Axial Flux Permanent-Magnet Machine With AirCored Nonoverlapping Concentrated Stator Windings', IEEE Transactions on Industry Applications, vol. 44, no. 5, pp. 1495-1504, Sep. 2008, doi: 10.1109/TIA.2008.2002183.

[33] N. Bianchi and M. D. Pré, 'Use of the star of slots in designing fractional-slot single-layer synchronous motors', IEE Proceedings Electric Power Applications, vol. 153, no. 3, pp. 459-466, May 2006.

[34] F. Tourkhani and P. Viarouge, 'Accurate analytical model of winding losses in round Litz wire windings', IEEE Transactions on Magnetics, vol. 37, no. 1, pp. 538-543, Jan. 2001, doi: 10.1109/20.914375.

[35] Rong-Jie Wang and M. J. Kamper, 'Calculation of eddy current loss in axial field permanent-magnet machine with coreless stator', IEEE Transactions on Energy Conversion, vol. 19, no. 3, pp. 532-538, Sep. 2004, doi: 10.1109/TEC.2004.832043. 
[36] R. Dorget et al., 'Review on the Use of Superconducting Bulks for Magnetic Screening in Electrical Machines for Aircraft Applications', Materials, vol. 14, no. 11, Art. no. 11, Jan. 2021, doi: 10.3390/ma14112847.

[37] M. D. Ainslie and H. Fujishiro, 'Modelling of bulk superconductor magnetization', Supercond. Sci. Technol., vol. 28, no. 5, p. 053002, Mar. 2015, doi: 10.1088/0953-2048/28/5/053002.

[38] K. Berger, J. Leveque, D. Netter, B. Douine, and A. Rezzoug, 'Influence of Temperature and/or Field Dependences of the \$E-J\$ Power Law on Trapped Magnetic Field in Bulk YBaCuO', IEEE Transactions on Applied Superconductivity, vol. 17, no. 2, pp. 3028 3031, Jun. 2007, doi: 10.1109/TASC.2007.902095.

[39] M. D. Ainslie et al., 'Modelling and comparison of trapped fields in (RE)BCO bulk superconductors for activation using pulsed field magnetization', Supercond. Sci. Technol., vol. 27, no. 6, p. 065008, Apr. 2014, doi: 10.1088/0953-2048/27/6/065008.

[40] C. P. Bean, 'Magnetization of High-Field Superconductors', Rev. Mod. Phys., vol. 36, no. 1, pp. 31-39, Jan. 1964, doi: 10.1103/RevModPhys.36.31. 